\title{
Global Synchronization for Discrete-Time Stochastic Complex Networks With Randomly Occurred Nonlinearities and Mixed Time Delays
}

\author{
Zidong Wang, Senior Member, IEEE, Yao Wang, and Yurong Liu
}

\begin{abstract}
In this paper, the problem of stochastic synchronization analysis is investigated for a new array of coupled discretetime stochastic complex networks with randomly occurred nonlinearities (RONs) and time delays. The discrete-time complex networks under consideration are subject to: 1) stochastic nonlinearities that occur according to the Bernoulli distributed white noise sequences; 2) stochastic disturbances that enter the coupling term, the delayed coupling term as well as the overall network; and 3) time delays that include both the discrete and distributed ones. Note that the newly introduced RONs and the multiple stochastic disturbances can better reflect the dynamical behaviors of coupled complex networks whose information transmission process is affected by a noisy environment (e.g., internet-based control systems). By constructing a novel Lyapunov-like matrix functional, the idea of delay fractioning is applied to deal with the addressed synchronization analysis problem. By employing a combination of the linear matrix inequality (LMI) techniques, the free-weighting matrix method and stochastic analysis theories, several delay-dependent sufficient conditions are obtained which ensure the asymptotic synchronization in the mean square sense for the discrete-time stochastic complex networks with time delays. The criteria derived are characterized in terms of LMIs whose solution can be solved by utilizing the standard numerical software. A simulation example is presented to show the effectiveness and applicability of the proposed results.
\end{abstract}

Index Terms-Discrete time delays, distributed time delays, global synchronization, randomly occurred nonlinearity (RON), stochastic complex networks, stochastic coupling.

\section{INTRODUCTION}

$\mathbf{O}$ VER the past few years, complex networks have been gaining increasing research attention because of their potential applications in many real-world systems from a variety

Manuscript received January 27, 2009; revised September 16, 2009; accepted September 24, 2009. First published December 01, 2009; current version published January 04, 2010. This work was supported in part by the Engineering and Physical Sciences Research Council (EPSRC) of the U.K. under Grant GR/S27658/01, an International Joint Project sponsored by the Royal Society of the U.K., the National Natural Science Foundation of China under Grant 60774073, the National 973 Program of China under Grant 2009CB320600, and the Alexander von Humboldt Foundation of Germany.

Z. Wang is with the School of Information Science and Technology, Donghua University, Shanghai 200051, China and also with the Department of Information Systems and Computing, Brunel University, Uxbridge, Middlesex UB8 3PH, U.K. (e-mail: Zidong.Wang@brunel.ac.uk).

Y. Wang is with the School of Information Science and Technology, Donghua University, Shanghai 200051, China (e-mail: enidyao@gmail.com).

Y. Liu is with the Department of Mathematics, Yangzhou University, Yangzhou 225002, China (e-mail: liuyurong@ gmail.com).

Color versions of one or more of the figures in this paper are available online at http://ieeexplore.ieee.org.

Digital Object Identifier 10.1109/TNN.2009.2033599 of fields such as biology, social systems, linguistic networks, and technological systems [1], [6], [13], [14], [18], [25], [29], [31], [32]. In particular, rapidly growing research results have been reported in the literature that have focused on the structural properties between the coupled nodes in the complex networks such as stability and robustness, see [14], [19], [38] for some recent publications. As one of the mostly investigated dynamical behaviors, the synchronization in complex networks with or without time delays has drawn significant research interest in recent years; see, e.g., [13]-[21], [25], [34] and the references therein.

The time-delay phenomenon in spreading information through complex networks is well known to be ubiquitous in nature, technology, and society because of the finite speed of signal transmission over the links as well as the network traffic congestions. Hence, constant or time-varying discrete delays have been considered in many existing results about the synchronization problem for complex (neural) networks; see, e.g., [15] and [25]. It is worth mentioning that, as a particular kind of time delays, the continuously distributed time delays have also received much research attention since a network usually has a spatial nature due to the presence of an amount of parallel pathways of a variety of axon sizes and lengths [17]-[19], [22] Accordingly, the synchronization problem for continuous-time complex networks with discrete and/or distributed time delays has been extensively investigated in [17] and [18]. Note that the corresponding results for the discrete-time complex networks with distributed time delays in the discrete time domain have been very few; see [19] for some initial research.

In real-time systems, the complex networks are often subject to noisy environment and, therefore, the stochastic modeling issue has been of vital importance in many branches of science such as neurotransmitters and network packet dropouts. In order to reflect more realistic dynamical behaviors, many researchers have recently investigated the problems of stochastic coupling and/or external stochastic disturbances for the synchronization of stochastic complex/neural networks [15], [28], [37]. It should be pointed out that, another interesting random phenomenon, namely, randomly switching connections, has been paid considerable research attention in the literature. For example, in [3], the global synchronization problem has been studied for continuous-time network with the so-called blinking connections that are randomly switched on and off with a given probability, and the frequency of switching is high compared to the network dynamics. In [26], the synchronization problem has been examined where the communication network topology changes ran- 
domly and is dictated by the agents' locations in the lattice. A stochastic genetic toggle switch model has been investigated in [27] where different time delays for transcription and translation and all reaction constant rates are randomly chosen from a range of values. In [9], it has been found that, for ensembles of yeast transcriptional network, those with deterministic Boolean rules are remarkably stable and those with random Boolean rules are only marginally stable. For various complex/neural/biological networks with deterministic switching topologies, we refer the readers to [2], [5], [30], and [35] for some representative publications.

Motivated by the rich literature on complex network with switching structures, in this paper, we aim to specifically address the randomly occurred nonlinearity (RON) that is an important phenomenon for the "blinking" networks discussed previously. As is well known, a wide class of practical systems are influenced by additive nonlinear disturbances that are caused by environmental circumstances. For complex networks with communication constraints, such nonlinear disturbances themselves may experience random abrupt changes, which may result from abrupt phenomena such as random failures and repairs of the components. Let us choose the networked systems and biological networks to justify the need of studying RONs. In a real-time networked environment, due to the limited bandwidth, network-induced packet losses, congestions, as well as quantization could be interpreted as a kind of external disturbances that occur in a probabilistic way and are randomly changeable in terms of their types and/or intensity. In a neural network, the signal transmission could be perturbed randomly from the release of probabilistic causes such as neurotransmitters. The randomly perturbed signals are in the form of spikes and most of the interaction with the other neurons takes place during the arrival of the spikes at the connection points, the synapses, which gives rise to a randomly switching interaction that is normally nonlinear. The so-called RONS, also called stochastic nonlinearities, have recently received some interest [33], and the filtering problem for discrete-time systems with stochastic nonlinearities has been thoroughly investigated. Nevertheless, to the best of the authors' knowledge, the synchronization problem for complex networks with specified RONs has not been fully investigated, and the purpose of this paper is therefore to shorten such a gap.

In this paper, the synchronization problem is studied for stochastic delayed discrete-time complex networks (SDDCN) with RONs, multiple stochastic disturbances, and mixed time delays. The proposed complex network model possesses the following characteristics: 1) the RONs described by the binary randomly switching sequences; 2) the distributed delay represented in the discrete-time setting; and 3) the multiple stochastic disturbances entering all coupling terms. The techniques we adopt are up to date in order to achieve delay-dependence for reducing possible conservatism. Specifically, we are interested in deriving sufficient conditions for the addressed problem by employing the properties of Kronecker product [12], the free-weighting approach [7], [8], and the stochastic analysis techniques [10], [23], combined with the "delay fractioning" approach [24], [25], [38]. A novel matrix functional is constructed to attain new synchronization criteria, which are formulated in the form of linear ma- trix inequalities (LMIs) [4]. Note that the LMIs can be solved by using the standard numerical software.

The remainder of this paper is organized as follows. In Section II, a stochastic discrete-time complex network model with mixed time delays, stochastic nonlinearities, and multiple stochastic disturbances is proposed, and some preliminaries are briefly outlined. In Section III, by utilizing the approach of "delay fractioning" and the matrix functional method, we conduct the stochastic analysis to obtain delay-dependent sufficient criteria in terms of LMIs so as to ensure that the addressed stochastic complex network to be globally synchronized in the mean square. In Section IV, a numerical example is provided to show the applicability of the obtained results. The conclusions are finally drawn in Section V.

Notations: Throughout this paper, $\mathbb{R}^{n}$ and $\mathbb{R}^{n \times m}$ denote, respectively, the $n$-dimensional Euclidean space and the set of all $n \times m$ real matrices. $P>0$ means that matrix $P$ is real, symmetric, and positive definite. $I$ and 0 denote the identity matrix and the zero matrix with compatible dimensions, respectively. $\operatorname{diag}\{\cdots\}$ stands for a block-diagonal matrix. $\operatorname{col}\{\cdots\}$ denotes a matrix column with blocks given by the matrices in $\{\cdots\}$. If $A$ is a matrix, denote by $\|A\|$ its operator norm, i.e., $\|A\|=\sup \{|A x|:|x|=1\}=\sqrt{\lambda_{\max }\left(A^{T} A\right)}$ where $\lambda_{\max }(\cdot)$ means the largest eigenvalue of $A$, and $|\cdot|$ is the Euclidean norm on $\mathbb{R}^{n}$. The superscript " $T$ " stands for matrix transposition and the asterisk "*" in a matrix is used to represent the term which is induced by symmetry. The Kronecker product of matrices $Q \in \mathbb{R}^{m \times n}$ and $R \in \mathbb{R}^{p \times q}$ is a matrix in $\mathbb{R}^{m p \times n q}$ and denoted as $Q \otimes R$. $\operatorname{col}\left\{M_{1}, M_{2}, \ldots, M_{n}\right\}$ stands for a column vector with its entries being $M_{1}, M_{2}, \ldots, M_{n}$. Moreover, let $\left(\Omega, \mathcal{F},\left\{\mathcal{F}_{t}\right\}_{t \geq 0}, P\right)$ be a complete probability space with a filtration $\left\{\mathcal{F}_{t}\right\}_{t \geq 0}$ satisfying the usual conditions (i.e., the filtration contains all $P$-null sets and is right continuous). $\mathbb{E}\{\cdot\}$ stands for the mathematical expectation operator with respect to the given probability measure $P$. Sometimes, the arguments of a function will be omitted in the analysis when no confusion arises.

\section{Problem Formulation AND PRELIMINARIES}

Consider the following array of SDDCN model consisting of $N$ coupled nodes of the form:

$$
\begin{aligned}
x_{m}(k+1)= & A x_{m}(k)+A_{d} x_{m}(k-d(k))+\gamma(k) B f\left(x_{m}(k)\right) \\
& +\vartheta(k) C g\left(x_{m}(k-d(k))\right) \\
& +\sum_{\beta=1}^{+\infty} \mu_{\beta} h\left(x_{m}(k-\beta)\right) \\
& +\sum_{\alpha=1}^{N} w_{m \alpha}^{(1)}\left(\Gamma_{1}+\omega_{1}(k) I\right) x_{\alpha}(k) \\
& +\sum_{\alpha=1}^{N} w_{m \alpha}^{(2)}\left(\Gamma_{2}+\omega_{2}(k) I\right) x_{\alpha}(k-d(k)) \\
& +\sigma_{m}\left(k, x_{m}(k), x_{m}(k-d(k))\right) \omega_{3}(k), \\
& m=1,2, \ldots, N
\end{aligned}
$$

where, for $k \in \mathbb{N}, x_{m}(k)=\operatorname{col}\left\{x_{m 1}(k), x_{m 2}(k)\right.$, $\left.\ldots, x_{m n}(k)\right\} \in \mathbb{R}^{n}$ is the state vector of the $m$ th node 
at time $k$ and $n$ denotes the number of nodes in each subsystem. $A, A_{d}, B$, and $C$ are known real matrices. $f\left(x_{m}(k)\right)=\operatorname{col}\left\{f_{1}\left(x_{m 1}(k)\right), f_{2}\left(x_{m 2}(k)\right), \ldots, f_{n}\left(x_{m n}(k)\right)\right\}$, $g\left(x_{m}(k-d(k))\right)=\operatorname{col}\left\{g_{1}\left(x_{m 1}(k-d(k))\right), g_{2}\left(x_{m 2}(k-\right.\right.$ $\left.d(k))), \ldots, g_{n}\left(x_{m n}(k-d(k))\right)\right\}$, and $h\left(x_{m}(k)\right)=$ $\operatorname{col}\left\{h_{1}\left(x_{m 1}(k)\right), h_{2}\left(x_{m 2}(k)\right), \ldots, h_{n}\left(x_{m n}(k)\right)\right\} \quad$ are nonlinear vector-valued functions satisfying certain conditions to be given later. The positive integer $d(k)$ describes the discrete time-varying delay that satisfies

$$
d_{m} \leq d(k) \leq d_{M}
$$

where $d_{m}, d_{M}$ are known positive integers representing the minimum and maximum delays, respectively.

Define the following random events for the system (1):

$$
\left\{\begin{array}{c}
\text { Event 1: System }(1) \text { experiences } \\
\text { nonlinear disturbance } f(\cdot) \\
\text { Event 2: System (1) experiences } \\
\text { nonlinear disturbance } g(\cdot) .
\end{array}\right.
$$

Then, the stochastic variables $\gamma(k) \in \mathbb{R}$ and $\vartheta(k) \in \mathbb{R}$ are given by the following two independent Bernoulli distributed white noise sequences:

$$
\gamma(k)=\left\{\begin{array}{l}
1: \text { if Event } 1 \text { occurs } \\
0: \text { if Event } 1 \text { does not occur }
\end{array}\right.
$$

and

$$
\vartheta(k)=\left\{\begin{array}{l}
1: \text { if Event } 2 \text { occurs } \\
0: \text { if Event } 2 \text { does not occur. }
\end{array}\right.
$$

Let $\gamma(k)$ and $\vartheta(k)$ satisfy the following distribution law:

$$
\begin{aligned}
& \operatorname{Prob}\{\gamma(k)=1\}=\mathbb{E}\{\gamma(k)\}=\gamma \\
& \operatorname{Prob}\{\gamma(k)=0\}=1-\mathbb{E}\{\gamma(k)\}=1-\gamma \\
& \operatorname{Prob}\{\vartheta(k)=1\}=\mathbb{E}\{\vartheta(k)\}=\theta \\
& \operatorname{Prob}\{\vartheta(k)=0\}=1-\mathbb{E}\{\vartheta(k)\}=1-\theta
\end{aligned}
$$

where $\gamma, \theta \in[01]$ are known constants reflecting the occurrence probability of the different nonlinear functions. The constant $\mu_{\beta} \geq 0$ satisfies the following convergence conditions:

$$
\tilde{\mu}=\sum_{\beta=1}^{+\infty} \mu_{\beta}<+\infty \quad \breve{\mu}=\sum_{\beta=1}^{+\infty} \beta \mu_{\beta}<+\infty .
$$

$\Gamma_{1}, \Gamma_{2}$ are inner-coupling matrices linking the $\alpha$ th state variable at time $k$ and $k-d(k)$, respectively; and $W^{(i)}=\left(w_{m \alpha}^{(i)}\right) \in$ $\mathbb{R}^{N \times N}$ denotes the outer-coupling configuration matrix of the network with $w_{m \alpha}^{(i)} \geq 0(m \neq \alpha)$ but not all zero, and the coupling configuration matrix $W^{(i)}$ is assumed to satisfy the diffusive connections

$$
\begin{aligned}
w_{m \alpha}^{(i)} & =w_{\alpha m}^{(i)}, \quad m \neq \alpha \\
\sum_{m=1}^{N} w_{m \alpha}^{(i)} & =0, \quad i=1,2, \quad m, \alpha=1,2, \ldots, N .
\end{aligned}
$$

Furthermore, we assume that $W^{(1)}$ and $W^{(2)}$ are commutative, i.e., $W^{(1)} W^{(2)}=W^{(2)} W^{(1)}$. Throughout this paper, the notations of $w_{m \alpha}^{(i)}$ and $w_{m \alpha}^{(i, j)}$ are used to denote the $(m, \alpha)$ entry of the matrix $W^{(i)}$ and $W^{(i)} W^{(j)}(i, j=1,2)$, respectively. Moreover, $\sigma_{m}(\cdot, \cdot, \cdot): \mathbb{R} \times \mathbb{R}^{n} \times \mathbb{R}^{n} \rightarrow \mathbb{R}^{n}$ is the noise intensity function vector; $\omega_{q}(k)(q=1,2,3)$ are scalar Wiener process (Brownian motion) defined on $(\Omega, \mathcal{F}, \mathcal{P})$ with

$$
\begin{array}{ll}
\mathbb{E}\left\{\omega_{q}(k)\right\}=0 & \mathbb{E}\left\{\omega_{p}(k) \omega_{q}(k)\right\}=0, \quad p \neq q \\
\mathbb{E}\left\{\omega_{q}^{2}(k)\right\}=1 & \mathbb{E}\left\{\omega_{q}(i) \omega_{q}(j)\right\}=0, \quad i \neq j
\end{array}
$$

where $\omega_{1}(k)$ and $\omega_{2}(k)$ are the stochastic coupling disturbances while $\omega_{3}(k)$ represents the system noise. Also, we should further assume that $\omega_{q}(k)(q=1,2,3)$ are independent with $\gamma(k)$ and $\vartheta(k)$.

Remark 1: It follows from the given hypothesis that $\mathbb{E}\{\gamma(k)$ $\gamma\}=0, \mathbb{E}\{\vartheta(k)-\theta\}=0, \mathbb{E}\left\{(\gamma(k)-\gamma)^{2}\right\}=\gamma(1-\gamma)$, and $\mathbb{E}\left\{(\vartheta(k)-\theta)^{2}\right\}=\theta(1-\theta)$. As pointed out in [36], $\gamma(k)$ and $\vartheta(k)$ are Markovian processes, which follow unknown but exponential distribution of switching.

Remark 2: In this paper, the random variables $\gamma(k)$ and $\vartheta(k)$ are used to model the probability distribution of the nonlinear functions. To our knowledge, this represents the first attempt to take into account the occurrence of different nonlinear functions in a probabilistic way for the addressed complex networks. In other words, in the complex network (1), the two terms $\gamma(k) B f\left(x_{i}(k)\right)$ and $\vartheta(k) C g\left(x_{i}(k-d(k))\right)$ can be used to account for the binary occurrence of these two nonlinear functions according to the given probability distribution.

Remark 3: The model of SDDCN (1) can be regarded as a discrete analog of the stochastic complex network in the continuous-time domain described by

$$
\begin{aligned}
& d x_{m}(t)=\left[A x_{m}(t)+A_{d} x_{m}(t-\tau(t))+\gamma(t) B f\left(x_{m}(t)\right)\right. \\
& +\vartheta(t) C g\left(x_{m}(t-\tau(t))\right) \\
& \left.+C \int_{-\infty}^{t} \mu(t-\beta) h\left(x_{m}(\beta)\right) d \beta\right] d t \\
& +\sum_{\alpha=1}^{N} w_{m \alpha}^{(1)} \Gamma_{1} x_{\alpha}(t)\left(d t+d \omega_{\alpha}(t)\right) \\
& +\sum_{\alpha=1}^{N} w_{m \alpha}^{(2)} \Gamma_{2} x_{\alpha}(t-\tau(t))\left(d t+d \omega_{2}(t)\right) \\
& +\sigma_{m}\left(t, x_{m}(t), x_{m}(t-\tau(t))\right) d \omega_{3}(t), \\
& m=1,2, \ldots, N \text {. }
\end{aligned}
$$

Throughout this paper, the following assumptions are made.

Assumption 1 [11]: For $\forall u, v \in \mathbb{R}^{n}$, the nonlinear functions $f(\cdot), g(\cdot)$, and $h(\cdot)$ are continuous and assumed to satisfy the following sector-bounded nonlinearity conditions:

$$
\begin{array}{r}
{\left[f(u)-f(v)-F_{1}(u-v)\right]^{T}\left[f(u)-f(v)-F_{2}(u-v)\right] \leq 0} \\
{\left[g(u)-g(v)-G_{1}(u-v)\right]^{T}\left[g(u)-g(v)-G_{2}(u-v)\right] \leq 0} \\
{\left[h(u)-h(v)-H_{1}(u-v)\right]^{T}\left[h(u)-h(v)-H_{2}(u-v)\right] \leq 0}
\end{array}
$$

where $F_{1}, G_{1}, H_{1}$ and $F_{2}, G_{2}, H_{2}$ are known constant real matrices. 
Remark 4: The description of nonlinear functions is known as the sector-like condition, which is more general than the commonly used Lipschitz conditions. Such a general description has been exploited in a number of papers, e.g., [18] and [19].

Assumption 2: The noise intensity function vector $\sigma_{i}: \mathbb{R} \times$ $\mathbb{R}^{n} \times \mathbb{R}^{n} \rightarrow \mathbb{R}^{n}$ satisfies the Lipschitz condition, i.e., there exist constant matrices $\mathcal{N}_{1}$ and $\mathcal{N}_{2}$ of appropriate dimensions such that the following inequality:

$\left|\sigma_{i}\left(k, u_{1}, v_{1}\right)-\sigma_{j}\left(k, u_{2}, v_{2}\right)\right|^{2} \leq\left|\mathcal{N}_{1}\left(u_{1}-u_{2}\right)\right|^{2}+\left|\mathcal{N}_{2}\left(v_{1}-v_{2}\right)\right|^{2}$

holds for all $i, j=1,2, \ldots, N$ and $u_{1}, v_{1}, u_{2}, v_{2} \in \mathbb{R}^{n}$.

For simplicity, we denote

$$
\begin{gathered}
x(k)=\operatorname{col}\left\{x_{1}(k), x_{2}(k), \ldots, x_{N}(k)\right\} \\
F(x(k))=\operatorname{col}\left\{f\left(x_{1}(k)\right), f\left(x_{2}(k)\right),\right. \\
\left.\ldots, f\left(x_{N}(k)\right)\right\} \\
H(x(k))=\operatorname{col}\left\{h\left(x_{1}(k)\right), h\left(x_{2}(k)\right),\right. \\
\left.\ldots, h\left(x_{N}(k)\right)\right\} \\
G(x(k-d(k)))=\operatorname{col}\left\{g\left(x_{1}(k-d(k))\right),\right. \\
g\left(x_{2}(k-d(k))\right), \\
\left.\ldots, g\left(x_{N}(k-d(k))\right)\right\} \\
\sigma(k, x(k), x(k-d(k)))=\operatorname{col}\left\{\sigma_{1}\left(k, x_{1}(k), x_{1}(k-d(k))\right),\right. \\
\sigma_{2}\left(k, x_{2}(k), x_{2}(k-d(k))\right), \ldots, \\
\left.\sigma_{N}\left(k, x_{N}(k), x_{N}(k-d(k))\right)\right\} .
\end{gathered}
$$

Using the matrix Kronecker product " $\otimes$," we can recast the network (1) into a more compact form as

$$
\begin{aligned}
x(k+1)= & \left(I_{N} \otimes A+W^{(1)} \otimes \Gamma_{1}\right) x(k) \\
& +\left(I_{N} \otimes A_{d}+W^{(2)} \otimes \Gamma_{2}\right) x(k-d(k)) \\
& +\gamma(k)\left(I_{N} \otimes B\right) F(x(k)) \\
& +\vartheta(k)\left(I_{N} \otimes C\right) G(x(k-d(k))) \\
& +\sum_{\beta=1}^{+\infty} \mu_{\beta} H(x(k-\beta))+\left(W^{(1)} \otimes I\right) x(k) \omega_{1}(k) \\
& +\left(W^{(2)} \otimes I\right) x(k-d(k)) \omega_{2}(k) \\
& +\sigma(k, x(k), x(k-d(k))) \omega_{3}(k) .
\end{aligned}
$$

Definition 1: The discrete-time stochastic complex network (1) [or (11)] is said to be asymptotically synchronized in the mean square if, for the addressed discrete and distributed delays, the following holds:

$$
\lim _{k \rightarrow+\infty} \mathbb{E}\left\{\left|x_{m}(k)-x_{\alpha}(k)\right|^{2}\right\}=0, \quad 1 \leq m<\alpha \leq N .
$$

Furthermore, the complex network (1) [or (11)] is said to be exponentially synchronized in the mean square if, for any solution $x(k)$ of system (11), there exist two constants $0<\varepsilon<1$ and $\varrho>0$ such that, for a sufficiently large integer $\kappa>0$, the inequality

$$
\mathbb{E}\left\{\left|x_{m}(k)-x_{\alpha}(k)\right|^{2}\right\} \leq \varrho \varepsilon^{k}, \quad 1 \leq m<\alpha \leq N
$$

holds for all $k>\kappa$.
In the rest of this paper, we aim to provide the sufficient conditions for the stochastic synchronization problem between the nodes of a class of discrete-time stochastic complex network (1) [or (11)] with both discrete and distributed time delays. With the "delay-fractioning" method, we construct a novel matrix functional and develop an LMI approach to derive the criteria to ensure the addressed stochastic complex networks to be synchronized in the mean square.

\section{MAIN RESUlts AND PROOFS}

In this section, the mean square synchronization problem for the stochastic system (1) [or (11)] is investigated by utilizing the LMI techniques and free-weighting matrix method as well as the stochastic analysis theory.

First, the following lemmas are needed in the derivations of our main results.

Lemma 1 [19]: Let $M \in \mathbb{R}^{n \times n}$ be a positive-semidefinite matrix, $\mathbf{x}_{i} \in \mathbb{R}^{n}$ and $a_{i} \geq 0(i=1,2, \ldots)$. If the series concerned are convergent, the following inequality holds:

$$
\left(\sum_{i=1}^{+\infty} a_{i} \mathbf{x}_{i}\right)^{T} M\left(\sum_{i=1}^{+\infty} a_{i} \mathbf{x}_{i}\right) \leq\left(\sum_{i=1}^{+\infty} a_{i}\right) \sum_{i=1}^{+\infty} a_{i} \mathbf{x}_{i}^{T} M \mathbf{x}_{i} .
$$

Lemma 2 (Schur Complement): Given constant matrices $\Omega_{1}, \Omega_{2}, \Omega_{3}$ where $\Omega_{1}=\Omega_{1}^{T}$ and $\Omega_{2}>0$, then

if and only if

$$
\Omega_{1}+\Omega_{3}^{T} \Omega_{2}^{-1} \Omega_{3}<0
$$

$$
\left[\begin{array}{cc}
\Omega_{1} & \Omega_{3}^{T} \\
\Omega_{3} & -\Omega_{2}
\end{array}\right]<0 .
$$

Lemma 3 [12]: Let $a \in \mathbb{R}$ and $A, B, C, D$ be matrices with appropriate dimensions. Then, the following statements about Kronecker product are true:

1) $a(A \otimes B)=(a A) \otimes B=A \otimes(a B)$;

2) $(A \otimes B)^{T}=A^{T} \otimes B^{T}$;

3) $(A \otimes B)(C \otimes D)=(A C) \otimes(B D)$;

4) $A \otimes B \otimes C=(A \otimes B) \otimes C=A \otimes(B \otimes C)$;

5) $(A+B) \otimes(C+D)=A \otimes C+B \otimes C+A \otimes D+B \otimes D$.

Lemma 4: Let $\mathcal{U}=\left(\alpha_{i j}\right)_{N \times N}, P \in \mathbb{R}^{n \times n}, x=$ $\operatorname{col}\left\{x_{1}, x_{2}, \ldots, x_{N}\right\}$, and $y=\operatorname{col}\left\{y_{1}, y_{2}, \ldots, y_{N}\right\}$ with $x_{k}, y_{k} \in \mathbb{R}^{n}(k=1,2, \ldots, N)$. If $\mathcal{U}=\mathcal{U}^{T}$ and each row sum of $\mathcal{U}$ is zero, then

$$
x^{T}(\mathcal{U} \otimes P) y=-\sum_{1 \leq i<j \leq N} \alpha_{i j}\left(x_{i}-x_{j}\right) P\left(y_{i}-y_{j}\right) .
$$

Lemma 5: Let $A=\left(a_{i j}\right)_{m \times n}, B=\left(b_{i j}\right)_{n \times q}$, and $C=$ $\left(c_{i j}\right)_{m \times q}=A B$. If each column sum of $A$ is zero or each row sum of $B$ is zero, then each column sum or row sum of $C$ is zero.

Now, we are in the position to deal with the stochastic synchronization problem of network (11). First, let us introduce the approach of "delay fractioning," that is, an important step we take in this paper lies in that the lower bound $d_{m}$ of the time-varying delay $d(k)$ can be described as $d_{m}=r \rho$, where $r, \rho$ are positive integers and $r$ denotes the number of fractions. Then, we represent the time delay $d(k)$ by two parts: a constant part $r \rho$ and a time-varying part $h(k)$, i.e.,

$$
d(k)=r \rho+h(k), \quad 0 \leq h(k) \leq d_{M}-r \rho .
$$




\section{Denoting}

$$
y(k)=\mathcal{A} \delta(k)
$$

where

$$
\begin{gathered}
\delta(k)=\operatorname{col}\left\{\Upsilon(k), x(k-r \rho), x(k-d(k)), x\left(k-d_{M}\right),\right. \\
F(x(k)), G(x(k-d(k))), H(x(k)), \\
\left.\quad \sum_{\beta=1}^{+\infty} \mu_{\beta} H(x(k-\beta))\right\} \\
\Upsilon(k)=\operatorname{col}\{x(k), x(k-\rho), \ldots, x(k-(r-1) \rho)\} \\
\mathcal{A}=\left[\left(I_{N} \otimes A+W^{(1)} \otimes \Gamma_{1}\right), 0_{N n \times r N n},\right. \\
\left(I_{N} \otimes A_{d}+W^{(2)} \otimes \Gamma_{2}\right), 0_{N n \times N n}, \\
\left.\gamma\left(I_{N} \otimes B\right), \theta\left(I_{N} \otimes C\right), 0_{N n \times N n}, I_{N n}\right]
\end{gathered}
$$

then network (11) can be simplified as

$$
x(k+1)=y(k)+\mathcal{B}(k) \delta(k)+\sigma(k) \omega(k)
$$

where $\sigma(k)=\left[\left(W^{(1)} \otimes I\right) x(k),\left(W^{(2)} \otimes I\right) x(k-d(k))\right.$, $\sigma(k, x(k), x(k-d(k)))], \omega(k)=\operatorname{col}\left\{\omega_{1}(k), \omega_{2}(k), \omega_{3}(k)\right\}$, $\mathcal{B}(k)=\left[0_{N n \times(r+3) N n},(\gamma(k)-\gamma)\left(I_{N} \otimes B\right),(\vartheta(k)-\theta)\left(I_{N} \otimes\right.\right.$ $\left.C), 0_{N n \times 2 N n}\right]$.

Hereafter, we will establish our main criterion based on the LMI approach. For brevity of the following analysis or presentation, we denote

$$
\begin{aligned}
& \breve{F}_{1}=F_{1}^{T} F_{2}+F_{2}^{T} F_{1}, \breve{F}_{2}=F_{1}^{T}+F_{2}^{T} \\
& \breve{G}_{1}=G_{1}^{T} G_{2}+G_{2}^{T} G_{1}, \breve{G}_{2}=G_{1}^{T}+G_{2}^{T} \\
& \breve{H}_{1}=H_{1}^{T} H_{2}+H_{2}^{T} H_{1}, \breve{H}_{2}=H_{1}^{T}+H_{2}^{T} \\
& \mathbf{x}_{m \alpha}(k)=x_{m}(k)-x_{\alpha}(k) \\
& \mathbf{f}_{m \alpha}(k)=f\left(x_{m}(k)\right)-f\left(x_{\alpha}(k)\right) \\
& \Upsilon_{m \alpha}(k)=\operatorname{col}\left\{\mathbf{x}_{m \alpha}(k), \mathbf{x}_{m \alpha}(k-\rho), \ldots,\right. \\
&\left.\mathbf{x}_{m \alpha}(k-(r-1) \rho)\right\}
\end{aligned}
$$

$\mathbf{g}_{m \alpha}(k-d(k))=g\left(x_{m}(k-d(k))\right)-g\left(x_{\alpha}(k-d(k))\right)$

$$
\mathbf{h}_{m \alpha}(k)=h\left(x_{m}(k)\right)-h\left(x_{\alpha}(k)\right)
$$$$
\widehat{\mathbf{h}}_{m \alpha}(k)=\sum_{\beta=1}^{+\infty} \mu_{\beta} h\left(x_{m}(k-\beta)\right)-\sum_{\beta=1}^{+\infty} \mu_{\beta} h\left(x_{\alpha}(k-\beta)\right)
$$$$
\sigma_{m \alpha}(k)=\sigma_{m}\left(k, x_{m}(k), x_{m}(k-d(k))\right)
$$$$
\text { - } \sigma_{\alpha}\left(k, x_{\alpha}(k), x_{\alpha}(k-d(k))\right)
$$$$
\delta_{m \alpha}(k)=\operatorname{col}\left\{\Upsilon_{m \alpha}(k), \mathbf{x}_{m \alpha}\left(k-d_{m}\right)\right. \text {, }
$$$$
\mathbf{x}_{m \alpha}(k-d(k)), \mathbf{x}_{m \alpha}\left(k-d_{M}\right) \text {, }
$$$$
\mathbf{f}_{m \alpha}(k), \mathbf{g}_{m \alpha}(k-d(k)) \text {, }
$$$$
\left.\mathbf{h}_{m \alpha}(k), \widehat{\mathbf{h}}_{m \alpha}(k)\right\} \text {. }
$$

Theorem 1: Under Assumptions 1 and 2, the stochastic complex network (11) is asymptotically mean square synchronized in the sense of Definition 1 if there exist positive-definite matrices $P_{i}(i=1,2), Q_{l}(l=1,2, \ldots, r), R, S, T$, real matrices $X_{j}, Y_{j}, Z_{j}(j=1,2, \ldots, r+7), M, J$, and four constants $\varrho_{l}>0(l=1,2,3), \lambda^{*}>0$ such that the following LMIs hold:

$$
\begin{aligned}
P_{1}+P_{2} & \leq \lambda^{*} I \\
\Pi_{m \alpha} & =\Pi_{1}+\Pi_{2}+\bar{\Pi}_{3}+\Pi_{4}+\Pi_{4}^{T}<0
\end{aligned}
$$

$$
\begin{aligned}
& \Xi_{1}=\left[\begin{array}{cc}
I_{r+7} \otimes M & \mathcal{X} \\
* & \hbar P_{2}
\end{array}\right] \geq 0 \\
& \Xi_{2}=\left[\begin{array}{cc}
I_{r+7} \otimes J & \mathcal{Y} \\
* & \hbar P_{2}
\end{array}\right] \geq 0 \\
& \Xi_{3}=\left[\begin{array}{cc}
I_{r+7} \otimes J & \mathcal{Z} \\
* & \hbar P_{2}
\end{array}\right] \geq 0
\end{aligned}
$$

where

$$
\begin{aligned}
& \Pi_{1}=\rho\left(I_{r+7} \otimes M\right)+\left(d_{M}-d_{m}\right)\left(I_{r+7} \otimes J\right) \\
& \Pi_{2}=W_{q}^{T} \mathcal{Q} W_{q} \quad \bar{\Pi}_{3}=W_{s}^{T} \Theta W_{s}
\end{aligned}
$$

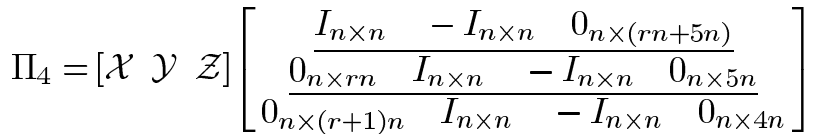$$
\mathcal{Q}=\left[\begin{array}{cc}
\bar{Q} & 0 \\
* & -\bar{Q}
\end{array}\right] \quad W_{q}=\left[\begin{array}{ccc}
I_{r n \times r n} & 0_{r n \times n} & 0_{r n \times 6 n} \\
0_{r n \times n} & I_{r n \times r n} & 0_{r n \times 6 n}
\end{array}\right]
$$$$
\bar{Q}=\operatorname{diag}\left\{Q_{1}, Q_{2}, \ldots, Q_{r}\right\}
$$

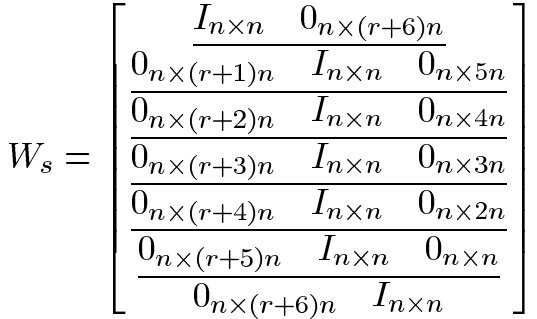

$$
\begin{aligned}
& \Theta=\left[\begin{array}{ccccccc}
\Theta_{11} & \Theta_{12} & 0 & \Theta_{14} & \Theta_{15} & \varrho_{3} \breve{H}_{2} & \Theta_{17} \\
* & \Theta_{22} & 0 & \Theta_{24} & \Theta_{25} & 0 & \Theta_{27} \\
* & * & -T & 0 & 0 & 0 & 0 \\
* & * & * & \Theta_{44} & \gamma \theta \bar{B} P \bar{C} & 0 & \gamma \bar{B} P \bar{I} \\
* & * & * & * & \Theta_{55} & 0 & \theta \bar{C} P \bar{I} \\
* & * & * & * & * & \Theta_{66} & 0 \\
* & * & * & * & * & * & \Theta_{77}
\end{array}\right]
\end{aligned}
$$$$
\Theta_{11}=\bar{A}^{T} P \bar{A}-N w_{m \alpha}^{(1,1)}\left[\bar{\Gamma}_{1}^{T} P \bar{\Gamma}_{1}+\bar{I}^{T} P \bar{I}\right]
$$$$
-N w_{m \alpha}^{(1)}\left[\bar{A}^{T} P \bar{\Gamma}_{1}+\bar{\Gamma}_{1}^{T} P \bar{A}\right]+\left(d_{M}-d_{m}+1\right) S
$$$$
+T+\lambda^{*} \mathcal{N}_{1}^{T} \mathcal{N}_{1}-P_{1}-\varrho_{1} \breve{F}_{1}-\varrho_{3} \breve{H}_{1}
$$$$
\Theta_{12}=\bar{A}^{T} P \bar{A}_{d}-N w_{m \alpha}^{(2)} \bar{A}^{T} P \bar{\Gamma}_{2}-N w_{m \alpha}^{(1)} \bar{\Gamma}_{1}^{T} P \bar{A}_{d}
$$$$
-N w_{m \alpha}^{(1,2)} \bar{\Gamma}_{1}^{T} P \bar{\Gamma}_{2}
$$$$
\Theta_{14}=\gamma\left[\bar{A} P \bar{B}-N w_{m \alpha}^{(1)} \bar{\Gamma}_{1}^{T} P \bar{B}\right]+\varrho_{1} \breve{F}_{2}
$$$$
\Theta_{15}=\theta\left[\bar{A} P \bar{C}-N w_{m \alpha}^{(1)} \bar{\Gamma}_{1}^{T} P \bar{C}\right]
$$$$
\Theta_{22}={\overline{A_{d}}}^{T} P \bar{A}_{d}-N w_{m \alpha}^{(2,2)}\left[\bar{\Gamma}_{2}^{T} P \bar{\Gamma}_{2}+\bar{I}^{T} P \bar{I}\right]
$$$$
-N w_{m \alpha}^{(2)}\left[\bar{A}_{d}^{T} P \bar{\Gamma}_{2}+\bar{\Gamma}_{2}^{T} P \bar{A}_{d}\right]
$$$$
+\lambda^{*} \mathcal{N}_{2}^{T} \mathcal{N}_{2}-S-\varrho_{2} \breve{G}_{1}
$$

$\Theta_{17}=\bar{A} P \bar{I}-N w_{m \alpha}^{(1)} \bar{\Gamma}_{1}^{T} P \bar{I}$

$\Theta_{24}=\gamma\left[\bar{A}_{d} P \bar{B}-N w_{m \alpha}^{(2)} \bar{\Gamma}_{2}^{T} P \bar{B}\right]$

$\Theta_{25}=\theta\left[\bar{A}_{d} P \bar{C}-N w_{m \alpha}^{(2)} \bar{\Gamma}_{2}^{T} P \bar{C}\right]+\varrho_{2} \breve{G}_{2}$

$\Theta_{27}=\bar{A}_{d} P \bar{I}-N w_{m \alpha}^{(2)} \bar{\Gamma}_{2}^{T} P \bar{I}$

$\Theta_{44}=\gamma \bar{B}^{T} P \bar{B}-2 \varrho_{1} I \quad \Theta_{55}=\theta \bar{C}^{T} P \bar{C}-2 \varrho_{2} I$

$\Theta_{66}=\tilde{\mu} R-2 \varrho_{3} I \quad \Theta_{77}=\bar{I}^{T} P \bar{I}-\frac{1}{\tilde{\mu}} R$

$P=\operatorname{diag}\left\{P_{1}, P_{2}\right\} \quad \bar{I}=\operatorname{col}\left\{I_{n}, I_{n}\right\}$

$\bar{\Gamma}_{i}=\operatorname{col}\left\{\Gamma_{i} \quad \Gamma_{i}\right\}, \quad i=1,2 \quad \bar{A}=\operatorname{col}\left\{A, A-I_{n}\right\}$

$\bar{A}_{d}=\operatorname{col}\left\{A_{d}, A_{d}\right\} \quad \bar{B}=\operatorname{col}\{B, B\} \quad \bar{C}=\operatorname{col}\{C, C\}$ 


$$
\begin{aligned}
& \mathcal{X}=\operatorname{col}\left\{X_{1}, X_{2}, \ldots, X_{r+7}\right\} \\
& \mathcal{Y}=\operatorname{col}\left\{Y_{1}, Y_{2}, \ldots, Y_{r+7}\right\} \\
& \mathcal{Z}=\operatorname{col}\left\{Z_{1}, Z_{2}, \ldots, Z_{r+7}\right\} .
\end{aligned}
$$

Proof: See the Appendix.

Remark 5: In the stochastic networks addressed in this paper, there are four main factors contributing to the complexities, namely, the RONs, discrete time delays, distributed time delays as well as stochastic disturbances on both the coupling and the overall networks. All these four factors have been explicitly reflected in the LMI conditions (15)-(17) given in Theorem 1; see the occurrence probabilities $\gamma$ and $\theta$ for the RONs, the minimum and maximum delays $d_{m}$ and $d_{M}$ for the discrete time delays, the "sum of the kernel information" $\tilde{\mu}$ for the distributed time delays, and the constant matrices $\mathcal{N}_{1}$ and $\mathcal{N}_{2}$ that quantify the noise intensities.

As a by-product of Theorem 1, we now reconsider the formulas (36)-(51) and obtain the following corollary by another calculation method.

Corollary 1: Under Assumptions 1 and 2, the stochastic complex network is asymptotically mean square synchronized in the sense of Definition 1 if there exist positive-definite matrices $P_{i}(i=1,2), Q_{l}(l=1,2, \ldots, r), R, S, T$, real matrices $X_{j}$, $Y_{j}, Z_{j}(j=1,2, \ldots, r+7)$, and four constants $\varrho_{l}>0(l=$ $1,2,3), \lambda^{*}>0$ such that the following LMIs hold:

$$
\left[\begin{array}{cccc}
\hat{\Pi}_{m \alpha} & \sqrt{\rho} \mathcal{X} & \sqrt{d_{M}-d_{m}} \mathcal{Y} & \sqrt{d_{M}-d_{m}} \mathcal{Z} \\
* & -\hbar P_{2} & 0 & 0 \\
* & * & -\hbar P_{2} & 0 \\
* & * & * & -\hbar P_{2}
\end{array}\right]<\lambda^{*} I
$$

where $\hat{\Pi}_{m \alpha}=\Pi_{2}+\bar{\Pi}_{3}+\Pi_{4}+\Pi_{4}^{T}$, and $\mathcal{X}, \mathcal{Y}$, and $\mathcal{Z}$ are defined in Theorem 1.

Proof: Similar to the proof of Theorem 1, here we just replace formula (52) by the following:

$$
\begin{aligned}
\mathbb{E}\{\Delta V(k)\} & \sum_{1 \leq m<\alpha \leq N} \mathbb{E}\left\{\begin{array}{c}
\delta_{m \alpha}^{T}(k)\left[\hat{\Pi}_{m \alpha}+\rho \mathcal{X}\left(\hbar P_{2}\right)^{-1} \mathcal{X}^{T}\right. \\
+\left(d_{M}-d_{m}\right) \mathcal{Y}\left(\hbar P_{2}\right)^{-1} \mathcal{Y}^{T} \\
\left.+\left(d_{M}-d_{m}\right) \mathcal{Z}\left(\hbar P_{2}\right)^{-1} \mathcal{Z}^{T}\right] \delta_{m \alpha}(k)
\end{array}\right. \\
& -\sum_{j=k-\rho}^{k-1}\left(\hbar P_{2} \eta_{m \alpha}(j)+\mathcal{X}^{T} \delta_{m \alpha}(k)\right)^{T} \\
& \times\left(\hbar P_{2}\right)^{-1}\left(\hbar P_{2} \eta_{m \alpha}(j)+\mathcal{X}^{T} \delta_{m \alpha}(k)\right) \\
& -\sum_{k-r \rho-1}\left(\hbar P_{2} \eta_{m \alpha}(j)+\mathcal{Y}^{T} \delta_{m \alpha}(k)\right)^{T} \\
& \times\left(\hbar P_{2}\right)^{-1}\left(\hbar P_{2} \eta_{m \alpha}(j)+\mathcal{Y}^{T} \delta_{m \alpha}(k)\right) \\
& -\sum_{k-r \rho-h(k)-1}\left(\hbar P_{2} \eta_{m \alpha}(j)+\mathcal{Z}^{T} \delta_{m \alpha}(k)\right)^{T} \\
& \left.\times\left(\hbar P_{2}\right)^{-1}\left(\hbar P_{2} \eta_{m \alpha}(j)+\mathcal{Z}^{T} \delta_{m \alpha}(k)\right)\right\} .
\end{aligned}
$$

Then, by Lemma 2, we know that LMI (19) guarantees

$$
\begin{aligned}
\hat{\Pi}_{m \alpha}+\rho \mathcal{X}\left(\hbar P_{2}\right)^{-1} \mathcal{X}^{T}+ & \left(d_{M}-d_{m}\right) \mathcal{Y}\left(\hbar P_{2}\right)^{-1} \mathcal{Y}^{T}+ \\
& \left(d_{M}-d_{m}\right) \mathcal{Z}\left(\hbar P_{2}\right)^{-1} \mathcal{Z}^{T}<0
\end{aligned}
$$

where $\hat{\Pi}_{m \alpha}=\Pi_{2}+\bar{\Pi}_{3}+\Pi_{4}+\Pi_{4}^{T}$, and $\mathcal{X}, \mathcal{Y}$ and $\mathcal{Z}$ are defined in (16). The rest of the proof follows the similar line in Theorem 1 , which is therefore omitted here.

To show that model (14) addressed in this paper is quite general, let us consider two special cases and the corresponding results are still believed to be new. To be concise, we omit the proofs that can easily follow from Theorem 1 .

Case 1: We first specialize system (14) to the case without RONs by setting $\gamma(k)=\gamma=1$ and $\vartheta(k)=\theta=1$ for all $k$. In this case, system (14) reduces to

$$
\begin{aligned}
x(k+1)= & \left(I_{N} \otimes A+W^{(1)} \otimes \Gamma_{1}\right) x(k) \\
& +\left(I_{N} \otimes A_{d}+W^{(2)} \otimes \Gamma_{2}\right) x(k-d(k)) \\
& +\left(I_{N} \otimes B\right) F(x(k))+\left(I_{N} \otimes C\right) G(x(k-d(k))) \\
& +\sum_{\beta=1}^{+\infty} \mu_{\beta} H(x(k-\beta))+\left(W^{(1)} \otimes I\right) x(k) \omega_{1}(k) \\
& +\left(W^{(2)} \otimes I\right) x(k-d(k)) \omega_{2}(k) \\
& +\sigma(k, x(k), x(k-d(k))) \omega_{3}(k) .
\end{aligned}
$$

The following result follows readily from Corollary 1 .

Corollary 2: Under Assumptions 1 and 2, the stochastic complex network is asymptotically mean square synchronized in the sense of Definition 1 if there exist positive-definite matrices $P_{i}(i=1,2), Q_{l}(l=1,2, \ldots, r), R, S, T$, real matrices $X_{j}$, $Y_{j}, Z_{j}(j=1,2, \ldots, r+7)$, and four constants $\varrho_{l}>0(l=$ $1,2,3), \lambda^{*}>0$ such that the following LMIs hold:

$$
\left[\begin{array}{cccc}
\breve{\Pi}_{m \alpha} & \sqrt{\rho} \mathcal{X} & \sqrt{d_{M}-d_{m}} \mathcal{Y} & \sqrt{d_{M}-d_{m}} \mathcal{Z} \\
* & -\hbar P_{2} & 0 & 0 \\
* & * & -\hbar P_{2} & 0 \\
* & * & * & -\hbar P_{2}
\end{array}\right]<\lambda^{*} I
$$

with $\breve{\Pi}_{m \alpha}=\Pi_{2}+\breve{\Pi}_{3}+\Pi_{4}+\Pi_{4}^{T}$. Here $\Pi_{2}, \Pi_{4}, \mathcal{X}, \mathcal{Y}$, and $\mathcal{Z}$ are defined in Theorem 1 , and $\breve{\Pi}_{3}$ is equal to $\bar{\Pi}_{3}$ with $\gamma=\theta=1$ in (16).

Case 2: Let us now assume that system (14) evolves with neither randomly occurred nonlinear functions nor multiple stochastic disturbances. In this case, (14) reduces to

$$
\begin{aligned}
x(k+1)= & \left(I_{N} \otimes A+W^{(1)} \otimes \Gamma_{1}\right) x(k) \\
& +\left(I_{N} \otimes A_{d}+W^{(2)} \otimes \Gamma_{2}\right) x(k-d(k)) \\
& +\left(I_{N} \otimes B\right) F(x(k))+\left(I_{N} \otimes C\right) G(x(k-d(k))) \\
& +\sum_{\beta=1}^{+\infty} \mu_{\beta} H(x(k-\beta)) .
\end{aligned}
$$

The following corollary is readily accessible from Theorem 1.

Corollary 3: Under Assumption 1, the complex network is asymptotically synchronized if there exist positive-definite matrices $P_{i}(i=1,2), Q_{l}(l=1,2, \ldots, r), R, S, T$, real matrices 
$X_{j}, Y_{j}, Z_{j}(j=1,2, \ldots, r+7), M, J$, and three constants $\varrho_{l}>0(l=1,2,3)$ such that the following LMIs hold:

$$
\begin{aligned}
\widetilde{\Pi}_{m \alpha} & =\Pi_{1}+\Pi_{2}+\widetilde{\Pi}_{3}+\Pi_{4}+\Pi_{4}^{T}<0 \\
\Xi_{1} & =\left[\begin{array}{cc}
I_{r+7} \otimes M & \mathcal{X} \\
* & \hbar P_{2}
\end{array}\right] \geq 0 \\
\Xi_{2} & =\left[\begin{array}{cc}
I_{r+7} \otimes J & \mathcal{Y} \\
* & \hbar P_{2}
\end{array}\right] \geq 0 \\
\Xi_{3} & =\left[\begin{array}{cc}
I_{r+7} \otimes J & \mathcal{Z} \\
* & \hbar P_{2}
\end{array}\right] \geq 0
\end{aligned}
$$

where $\Pi_{1}, \Pi_{2}, \Pi_{4}, \mathcal{X}, \mathcal{Y}$, and $\mathcal{Z}$ are defined in Theorem 1 , and $\widetilde{\Pi}_{3}=W_{s}^{T} \widetilde{\Theta} W_{s}$ with

$$
\begin{aligned}
& \widetilde{\Theta}=\left[\begin{array}{ccccccc}
\widetilde{\Theta}_{11} & \Theta_{12} & 0 & \widetilde{\Theta}_{14} & \widetilde{\Theta}_{15} & \varrho_{3} \breve{H}_{2} & \Theta_{17} \\
* & \widetilde{\Theta}_{22} & 0 & \widetilde{\Theta}_{24} & \widetilde{\Theta}_{25} & 0 & \Theta_{27} \\
* & * & -T & 0 & 0 & 0 & 0 \\
* & * & * & \widetilde{\Theta}_{44} & \bar{B} P \bar{C} & 0 & \bar{B} P \bar{I} \\
* & * & * & * & \widetilde{\Theta}_{55} & 0 & \bar{C} P \bar{I} \\
* & * & * & * & * & \Theta_{66} & 0 \\
* & * & * & * & * & * & \Theta_{77}
\end{array}\right] \\
& \widetilde{\Theta}_{11}=\bar{A}^{T} P \bar{A}-N w_{m \alpha}^{(1,1)} \bar{\Gamma}_{1}^{T} P \bar{\Gamma}_{1} \\
& -N w_{m \alpha}^{(1)}\left[\bar{A}^{T} P \bar{\Gamma}_{1}+\bar{\Gamma}_{1}^{T} P \bar{A}\right]+\left(d_{M}-d_{m}+1\right) S \\
& +T-P_{1}-\varrho_{1} \breve{F}_{1}-\varrho_{3} \breve{H}_{1} \\
& \widetilde{\Theta}_{14}=\bar{A} P \bar{B}-N w_{m \alpha}^{(1)} \bar{\Gamma}_{1}^{T} P \bar{B}+\varrho_{1} \breve{F}_{2} \\
& \widetilde{\Theta}_{15}=\bar{A} P \bar{C}-N w_{m \alpha}^{(1)} \bar{\Gamma}_{1}^{T} P \bar{C} \\
& \widetilde{\Theta}_{22}={\overline{A_{d}}}^{T} P \bar{A}_{d}-N w_{m \alpha}^{(2,2)} \bar{\Gamma}_{2}^{T} P \bar{\Gamma}_{2} \\
& -N w_{m \alpha}^{(2)}\left[\bar{A}_{d}^{T} P \bar{\Gamma}_{2}+\bar{\Gamma}_{2}^{T} P \bar{A}_{d}\right]-S-\varrho_{2} \breve{G}_{1} \\
& \widetilde{\Theta}_{24}=\overline{A_{d}} P \bar{B}-N w_{m \alpha}^{(2)} \bar{\Gamma}_{2}^{T} P \bar{B} \\
& \widetilde{\Theta}_{25}=\bar{A}_{d} P \bar{C}-N w_{m \alpha}^{(2)} \bar{\Gamma}_{2}^{T} P \bar{C}+\varrho_{2} \breve{G}_{2} \\
& \widetilde{\Theta}_{44}=\bar{B}^{T} P \bar{B}-2 \varrho_{1} I \quad \widetilde{\Theta}_{55}=\bar{C}^{T} P \bar{C}-2 \varrho_{2} I
\end{aligned}
$$

and the other symbols are the same as defined in Theorem 1.

Remark 6: Based on the general assumption of time-varying delay in the discrete-time system, we partition $d(k)$ by the timevarying part and the constant part. Thus, in case of the constant time delay, we can simply take $d_{M}=d_{m}$ and obtain the similar results by taking $S=0$ in the matrix functional (28).

Remark 7: In this paper, the random occurrence nonlinearity or stochastic nonlinearity is introduced by using binary randomly switching sequences to describe the distribution of nonlinear functions in a probabilistic way. Based on the setting in (14), we can see that the RONs have been divided into two parts, one of which is the deterministic functions $\gamma F(x(k))$ and $\theta G(x(k-d(k)))$, and the other one is the stochastic functions $(\gamma(k)-\gamma) F(x(k))$ and $(\vartheta(k)-\theta) G(x(k-d(k)))$. Moreover, it is not difficult to see that the mathematical expectation of the two terms in the stochastic part is zero. Hence, by adopting this conversion to deal with the stochastic nonlinearity, and using the stochastic differential equation theory, we can obtain our main results in that the probabilities of the corresponding randomly switching sequences are contained.

Remark 8: Novel delay-dependent criteria are established in both Theorem 1 and Corollary 1 for the synchroniza- tion problem of a new class discrete-time stochastic delayed complex networks. By introducing the "delay-fractioning" approach, we construct a novel matrix functional and utilize a combination of the free-weighting matrix method and the properties of Kronecker product. The utilization of the stochastic analysis technique results in the synchronization conditions expressed in terms of LMIs. Obviously, the size of LMIs grows as the node number increases. The LMI control toolbox implements state-of-the-art interior-point LMI solvers. While these solvers are significantly faster than classical convex optimization algorithms, it should be kept in mind that the complexity of LMI computations remains higher than that of solving, say, a Riccati equation. For instance, problems with a thousand design variables typically take over an hour on today's workstations. However, research on LMI optimization is a very active area in the applied math, optimization, and the operations research community, and substantial speedups can be expected in the future.

\section{NUMERICAL SimULATION}

In this section, a numerical example is presented to demonstrate the usefulness and applicability of the proposed testing criteria on the synchronization stability of the SDDCN (1) with mixed time delays.

Consider a SDDCN (1) with four nodes where the state vector of each node is 2-D, i.e., $N=4, n=2$. Other parameters in the example are given as follows:

$$
\begin{aligned}
A & =\left[\begin{array}{cc}
0.13 & -0.26 \\
0.31 & 0.42
\end{array}\right] \quad A_{d}=\left[\begin{array}{ll}
0.12 & 0.15 \\
0.32 & 0.21
\end{array}\right] \\
B & =\left[\begin{array}{ll}
0.25 & 0.75 \\
0.35 & 0.25
\end{array}\right] \quad C=\left[\begin{array}{ccc}
0.14 & 0.15 \\
0.45 & 0.23
\end{array}\right] \\
W^{(1)} & =W^{(2)}=0.15 \times\left[\begin{array}{cccc}
-3 & 1 & 1 & 1 \\
1 & -3 & 1 & 1 \\
1 & 1 & -3 & 1 \\
1 & 1 & 1 & -3
\end{array}\right] \\
\Gamma_{1} & =\Gamma_{2}=0.25 I_{2} \\
\mu_{\beta} & =2^{-(\beta+3)} \quad \gamma=0.58 \quad \theta=0.76 \\
d(k) & =4+\sin (k \pi / 4) .
\end{aligned}
$$

The noise diffusion coefficient vector satisfies Assumption 2 with

$$
\mathcal{N}_{1}=\left[\begin{array}{cc}
0.1 & -0.4 \\
-0.05 & 0.25
\end{array}\right] \quad \mathcal{N}_{2}=\left[\begin{array}{cc}
0.1 & 0.05 \\
-0.1 & 0.3
\end{array}\right]
$$

Let the nonlinear vector-valued functions be given by

$$
\begin{gathered}
f\left(x_{m}(k)\right)=\operatorname{col}\left\{-0.4 x_{m 1}(k)+0.3 x_{m 2}(k)\right. \\
+\tanh \left(0.3 x_{m 1}(k)\right), \\
\left.0.8 x_{m 2}(k)-\tanh \left(0.4 x_{m 2}(k)\right)\right\} \\
g\left(x_{m}(k)\right)=\operatorname{col}\left\{0.1 x_{m 2}(k)-\tanh \left(0.2 x_{m 1}(k)\right),\right. \\
\left.0.2 x_{m 2}(k)\right\} \\
h\left(x_{m}(k)\right)=\operatorname{col}\left\{0.3 x_{m 1}(k)-\tanh \left(0.1 x_{m 1}(k)\right),\right. \\
\left.0.1 x_{m 2}(k)\right\}, \quad m=1,2,3,4 .
\end{gathered}
$$

Then, it can be verified that $\tilde{\mu}=0.125$, and the lower bound and the upper bound of the discrete time delay are $d_{m}=3$ 

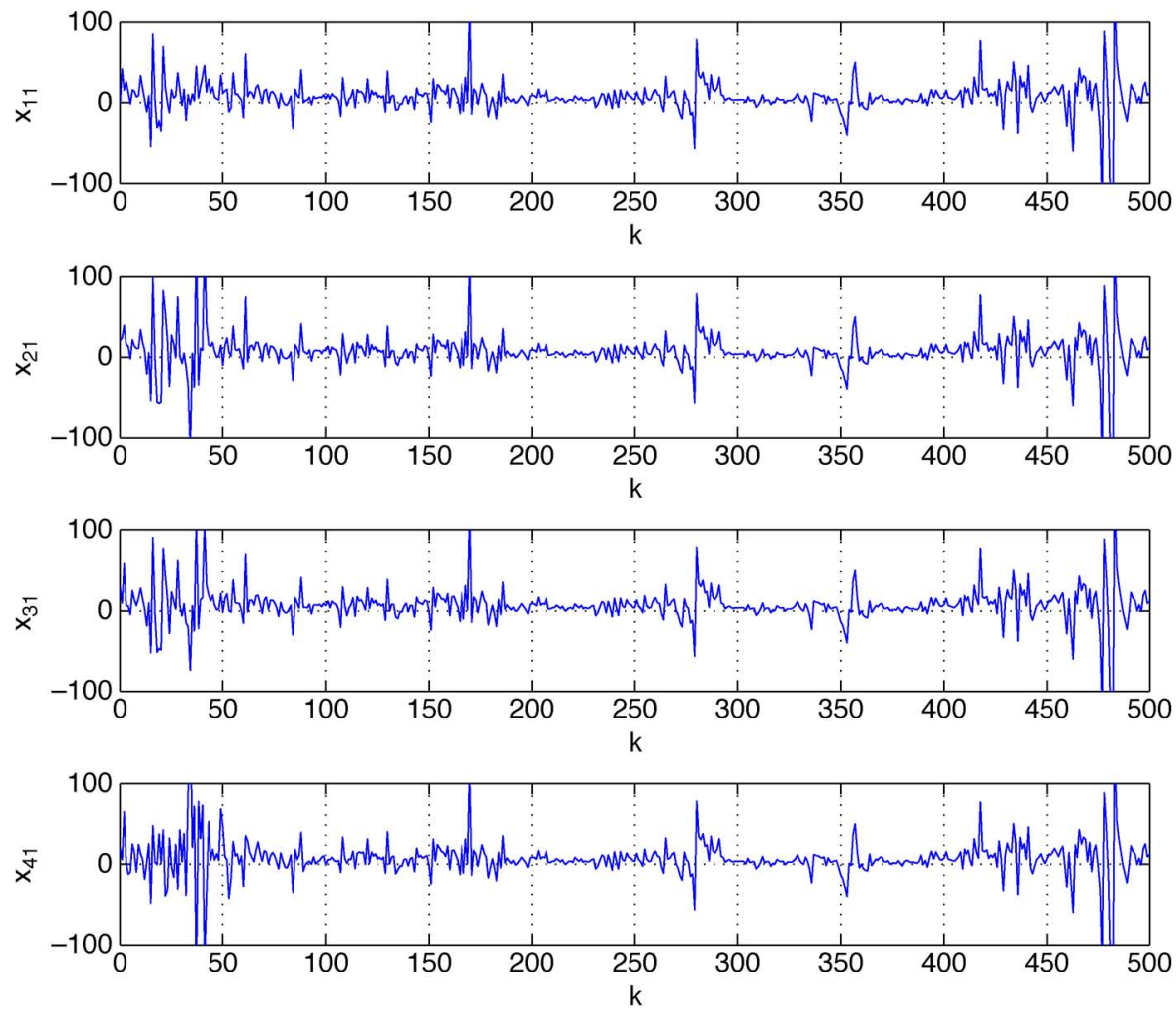

Fig. 1. State trajectories of $x_{i 1}(k)(i=1,2,3,4)$.
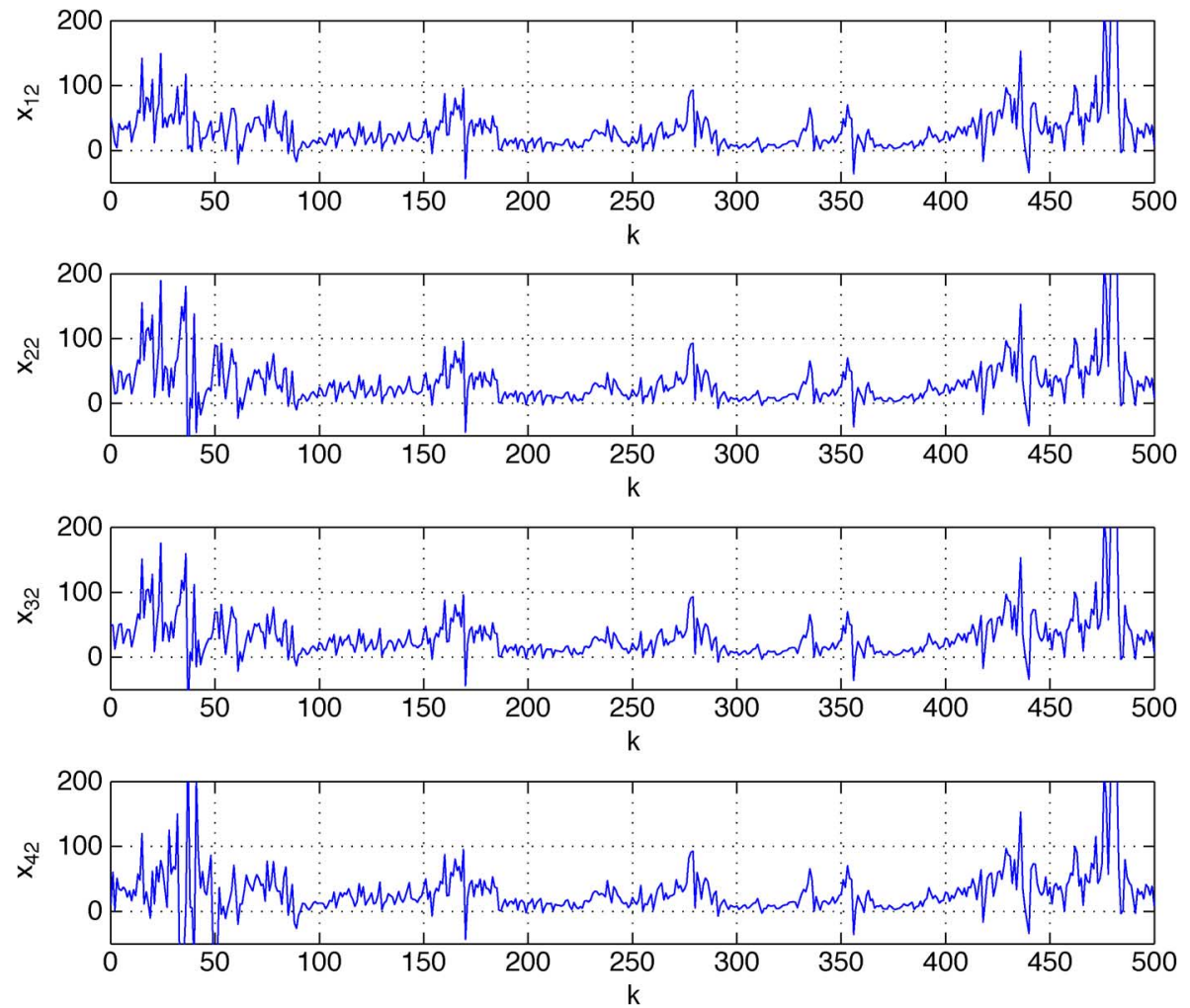

Fig. 2. State trajectories of $x_{i 2}(k)(i=1,2,3,4)$. 

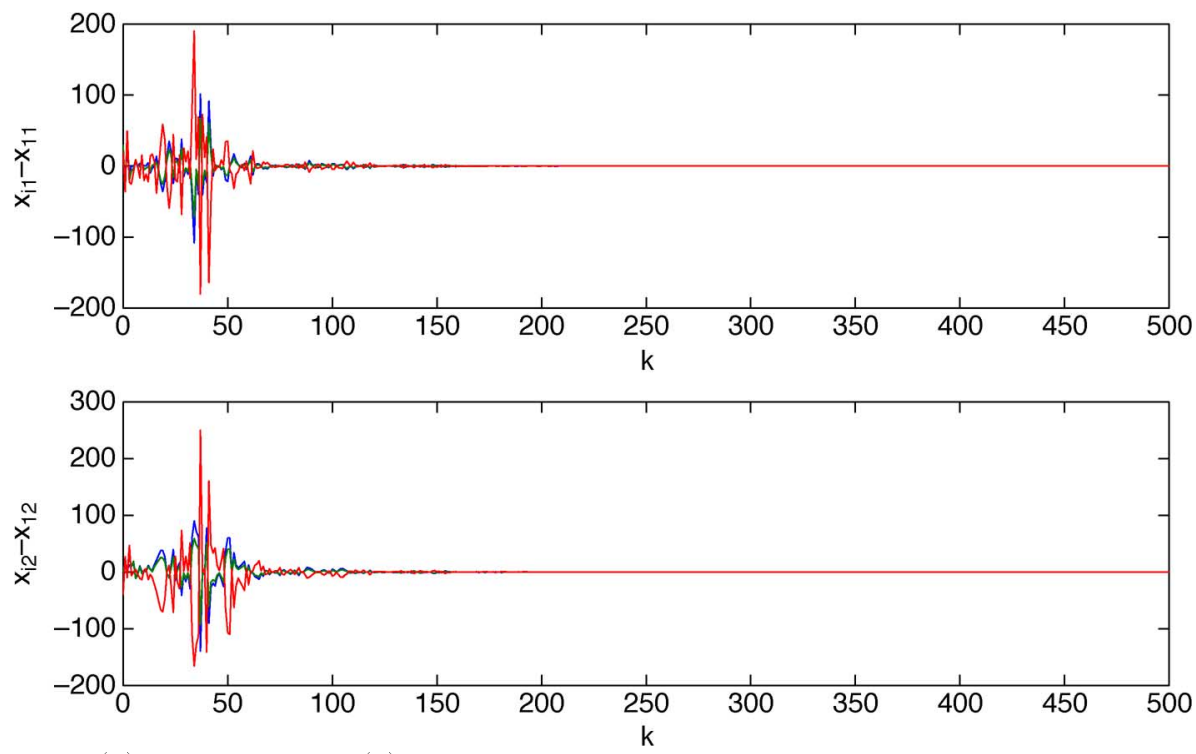

Fig. 3. Error states of between $x_{i}(k)(i=2,3,4)$ and $x_{1}(k)$.

and $d_{M}=5$, respectively. Also, it is not difficult to see that Assumption 1 is satisfied with

$$
\begin{aligned}
F_{1}=\left[\begin{array}{cc}
-0.4 & 0.3 \\
0 & 0.4
\end{array}\right] & F_{2}=\left[\begin{array}{cc}
-0.1 & 0.3 \\
0 & 0.8
\end{array}\right] \\
G_{1}=\left[\begin{array}{cc}
-0.2 & 0.1 \\
0 & 0.2
\end{array}\right] & G_{2}=\left[\begin{array}{ll}
0 & 0.1 \\
0 & 0.2
\end{array}\right] \\
H_{1}=\left[\begin{array}{cc}
0.2 & 0 \\
0 & 0.1
\end{array}\right] & H_{2}=\left[\begin{array}{cc}
0.3 & 0 \\
0 & 0.1
\end{array}\right] .
\end{aligned}
$$

Letting $r=1$ in the matrix functional (28) and using the Matlab LMI toolbox, we can find a feasible solution to the LMIs in (15)-(17) as follows:

$$
\begin{aligned}
P_{1} & =\left[\begin{array}{cc}
18.0252 & -3.3007 \\
-3.3007 & 16.3605
\end{array}\right] \quad P_{2}=\left[\begin{array}{ll}
2.0434 & 2.3683 \\
2.3683 & 7.6608
\end{array}\right] \\
Q_{1} & =\left[\begin{array}{cc}
3.9138 & -0.7122 \\
-0.7122 & 1.1288
\end{array}\right] \\
R & =\left[\begin{array}{cc}
8.1296 & 1.2843 \\
1.2843 & 19.0489
\end{array}\right] \quad S=\left[\begin{array}{cc}
0.3676 & -0.0895 \\
-0.0895 & 0.1000
\end{array}\right] \\
T & =\left[\begin{array}{cc}
6.8867 & -2.0014 \\
-2.0014 & 1.0701
\end{array}\right] \\
\lambda^{*} & =24.7570 \quad \varrho_{1}=13.6054 \quad \varrho_{2}=17.0223 \quad \varrho_{3}=19.6402 .
\end{aligned}
$$

According to Theorem 1, the array of coupled delayed complex networks (1) with multiple stochastic disturbances and stochastic nonlinearities can reach asymptotic synchronization in the mean square. The numerical simulation supports the theoretical results perfectly. Specially, in Figs. 1 and 2, we show the evolution of the state trajectories of each node $x_{i}(k)(i=$ $1,2,3,4)$. It is also noticed from Fig. 3 that the synchronization error between the states of the whole network approaches zero asymptotically.

Remark 9: It is worth pointing out that the example we designed in this section is nontrivial in evaluating the synchronization error since the synchronization analysis is investigated between all the unstable nodes in identical stochastic complex networks. In other words, the unstable complex networks are of more significance to be exploited to test and support the theoretical results.

\section{CONCLUSION}

In this paper, we have investigated the synchronization problem of a new array of coupled delayed complex networks with stochastic nonlinearities, multiple stochastic disturbances, and mixed time delays in the discrete-time domain. In order to study the stochastic nonlinearities for the addressed complex networks, we have first defined two sequences of random variables to model the probability distribution of the nonlinear functions. The description of the nonlinear functions is more general than the commonly used Lipschitz conditions. By utilizing a novel matrix functional, the properties of Kronecker product, the free-weighting matrix method, and the stochastic techniques, we have derived the synchronization stability criteria that are characterized in terms of LMIs, which can be readily checked by using standard numerical software. A simple example has been presented to show the effectiveness of the proposed results.

\section{APPENDIX}

\section{A. Proof of Theorem 1}

Based on the delay-fractioning idea, we conduct the following matrix functional:

$$
V(k)=\sum_{i=1}^{5} V_{i}(k)
$$

where

$$
\begin{aligned}
& V_{1}(k)=x^{T}(k)\left(U \otimes P_{1}\right) x(k) \\
& V_{2}(k)=\sum_{\beta=1}^{+\infty} \mu_{\beta} \sum_{j=k-\beta}^{k-1} H^{T}(x(j))(U \otimes R) H(x(j)) \\
& V_{3}(k)=\sum_{j=-d_{M}+1}^{-r \rho+1} \sum_{i=k-1+j}^{k-1} x^{T}(i)(U \otimes S) x(i)
\end{aligned}
$$




$$
\begin{aligned}
V_{4}(k)= & \sum_{i=k-\rho}^{k-1} \Upsilon^{T}(i) Q \Upsilon(i)+\sum_{i=k-d_{M}}^{k-1} x^{T}(i)(U \otimes T) x(i) \\
V_{5}(k)= & \sum_{i=-\rho}^{-1} \sum_{j=k+i}^{k-1} \eta^{T}(j)\left(U \otimes \hbar P_{2}\right) \eta(j) \\
& +\sum_{i=-d_{M}}^{-r \rho-1} \sum_{j=k+i}^{k-1} \eta^{T}(j)\left(U \otimes \hbar P_{2}\right) \eta(j) \\
Q= & \operatorname{diag}\left\{U \otimes Q_{1}, U \otimes Q_{2}, \ldots, U \otimes Q_{r}\right\} \\
\eta(j)= & x(j+1)-x(j) \quad \hbar=1 /\left(d_{M}-d_{m}+\rho\right) \\
\Upsilon(l)= & \operatorname{col}\{x(l) x(l-\rho) x(l-2 \rho) \ldots x(l-(r-1) \rho)\}
\end{aligned}
$$

and $U=\left(u_{m \alpha}\right)_{N \times N}$ with

$$
u_{m \alpha}= \begin{cases}-1, & \alpha \neq m \\ N-1, & \alpha=m\end{cases}
$$

From condition (6), it is not difficult to see that $V_{2}(k)$ in (28) is convergent. By calculating the difference of $V(k)$ along the solutions of network (11) and taking the mathematical expectation, we have

$$
\mathbb{E}\{\Delta V(k)\}=\sum_{i=1}^{5} \mathbb{E}\left\{\Delta V_{i}(k)\right\}
$$

where we have (30) shown at the bottom of the page and (31)-(34), shown on the next page, where $\eta(k)=$ $x(k+1)-x(k)=(\mathcal{A}-\mathcal{I}) \delta(k)+\mathcal{B}(k) \delta(k)+\sigma(k) \omega(k)$, with $\mathcal{I}=\left[I_{N} \otimes I_{n}, 0_{N n \times(r+6) N n}\right]$.

By virtue of Lemma 3, one can calculate $U W^{(i)}=W^{(i)} U=$ $N W^{(i)}(i=1,2)$, and

$$
\begin{aligned}
& \left(W^{(i)} \otimes \Gamma_{j}\right)^{T}(U \otimes P)\left(W^{(k)} \otimes \Gamma_{l}\right) \\
& \quad=\left(W^{(i)^{T}} \otimes \Gamma_{j}^{T}\right)(U \otimes P)\left(W^{(k)} \otimes \Gamma_{l}\right) \\
& =\left(W^{(i)^{T}} U W^{(k)}\right) \otimes\left(\Gamma_{j}^{T} P \Gamma_{l}\right) \\
& =N W^{(i)} W^{(k)} \otimes\left(\Gamma_{j}^{T} P \Gamma_{l}\right) \\
& \quad=: N W^{(i, k)} \otimes\left(\Gamma_{j}^{T} P \Gamma_{l}\right), \quad i, j, k, l=1,2 .
\end{aligned}
$$

$$
\begin{aligned}
& \mathbb{E}\left\{\Delta V_{1}(k)\right\} \\
& =\mathbb{E}\left\{V_{1}(k+1)-V_{1}(k)\right\}=\mathbb{E}\left\{x^{T}(k+1)\left(U \otimes P_{1}\right) x(k+1)-x^{T}(k)\left(U \otimes P_{1}\right) x(k)\right\} \\
& =\mathbb{E}\left\{x^{T}(k)\left(I_{N} \otimes A^{T}+\left(W^{(1)} \otimes \Gamma_{1}\right)^{T}\right)\left(U \otimes P_{1}\right)\right. \\
& \times\left[\left(I_{N} \otimes A+W^{(1)} \otimes \Gamma_{1}\right) x(k)\right. \\
& \left.+2\left(\left(I_{N} \otimes A_{d}+W^{(2)} \otimes \Gamma_{2}\right) x(k-d(k))+\left(\gamma I_{N} \otimes B\right) F(x(k))+\left(\theta I_{N} \otimes C\right) G(x(k-d(k)))+\sum_{\beta=1}^{+\infty} \mu_{\beta} H(x(k-\beta))\right)\right] \\
& +x^{T}(k-d(k))\left(I_{N} \otimes A_{d}+W^{(2)} \otimes \Gamma_{2}\right)^{T}\left(U \otimes P_{1}\right) \\
& \times\left[\left(I_{N} \otimes A_{d}+W^{(2)} \otimes \Gamma_{2}\right) x(k-d(k))+2\left(\left(\gamma I_{N} \otimes B\right) F(x(k))+\left(\theta I_{N} \otimes C\right) G(x(k-d(k)))+\sum_{\beta=1}^{+\infty} \mu_{\beta} H(x(k-\beta))\right)\right] \\
& +F^{T}(x(k))\left(\gamma I_{N} \otimes B^{T}\right)\left(U \otimes P_{1}\right)\left[\left(I_{N} \otimes B\right) F(x(k))+2\left(\theta I_{N} \otimes C\right) G(x(k-d(k)))+2 \sum_{\beta=1}^{+\infty} \mu_{\beta} H(x(k-\beta))\right] \\
& +G^{T}(x(k-d(k)))\left(\theta I_{N} \otimes C\right)^{T}\left(U \otimes P_{1}\right)\left[\left(I_{N} \otimes C\right) G(x(k-d(k)))+2 \sum_{\beta=1}^{+\infty} \mu_{\beta} H(x(k-\beta))\right] \\
& +\left(\sum_{\beta=1}^{+\infty} \mu_{\beta} H(x(k-\beta))\right)^{T}\left(U \otimes P_{1}\right)\left(\sum_{\beta=1}^{+\infty} \mu_{\beta} H(x(k-\beta))\right)+x^{T}(k)\left(W^{(1)} \otimes I\right)^{T} \\
& \times\left(U \otimes P_{1}\right)\left(W^{(1)} \otimes I\right) x(k)+x^{T}(k-d(k))\left(W^{(2)} \otimes I\right)^{T}\left(U \otimes P_{1}\right)\left(W^{(2)} \otimes I\right) x(k-d(k)) \\
& \left.+\sigma^{T}(k, x(k), x(k-d(k)))\left(U \otimes P_{1}\right) \sigma(k, x(k), x(k-d(k)))-x^{T}(k)\left(U \otimes P_{1}\right) x(k)\right\}
\end{aligned}
$$




$$
\begin{aligned}
\mathbb{E} & \left\{\Delta V_{2}(k)\right\} \\
& =\mathbb{E}\left\{V_{2}(k+1)-V_{2}(k)\right\} \\
& =\mathbb{E}\left\{\sum_{\beta=1}^{+\infty} \mu_{\beta}\left[\sum_{j=k+1-\beta}^{k} H^{T}(x(j))(U \otimes R) H(x(j))-\sum_{j=k-\beta}^{k-1} H^{T}(x(j))(U \otimes R) H(x(j))\right]\right\} \\
& =\mathbb{E}\left\{\sum_{\beta=1}^{+\infty} \mu_{\beta}\left[H^{T}(x(k))(U \otimes R) H(x(k))-H^{T}(x(k-\beta))(U \otimes R) H(x(k-\beta))\right]\right\} \\
& =\mathbb{E}\left\{\sum_{\beta=1}^{+\infty} \mu_{\beta} H^{T}(x(k))(U \otimes R) H(x(k))-\sum_{\beta=1}^{+\infty} \mu_{\beta} H^{T}(x(k-\beta))(U \otimes R) H(x(k-\beta))\right\} \\
& \leq \mathbb{E}\left\{\tilde{\mu} H^{T}(x(k))(U \otimes R) H(x(k))-\frac{1}{\tilde{\mu}}\left(\sum_{\beta=1}^{+\infty} \mu_{\beta} H(x(k-\beta))\right)^{T}(U \otimes R)\left(\sum_{\beta=1}^{+\infty} \mu_{\beta} H(x(k-\beta))\right)\right\}
\end{aligned}
$$

$$
\begin{aligned}
\mathbb{E} & \left\{\Delta V_{3}(k)\right\} \\
& =\mathbb{E}\left\{V_{3}(k+1)-V_{3}(k)\right\} \\
& =\mathbb{E}\left\{\sum_{j=-d_{M}+1}^{-r \rho+1}\left[\sum_{i=k+j}^{k} x^{T}(i)(U \otimes S) x(i)-\sum_{i=k-1+j}^{k-1} x^{T}(i)(U \otimes S) x(i)\right]\right\} \\
& =\mathbb{E}\left\{\left(d_{M}-r \rho+1\right) x^{T}(k)(U \otimes S) x(k)-\sum_{i=k-d_{M}}^{k-r \rho} x^{T}(i)(U \otimes S) x(i)\right\} \\
& \leq \mathbb{E}\left\{\left(d_{M}-r \rho+1\right) x^{T}(k)(U \otimes S) x(k)-x^{T}(k-d(k))(U \otimes S) x(k-d(k))\right\}
\end{aligned}
$$

$$
\begin{aligned}
\mathbb{E} & \left\{\Delta V_{4}(k)\right\} \\
& =\mathbb{E}\left\{V_{4}(k+1)-V_{4}(k)\right\} \\
& =\mathbb{E}\left\{\left[\sum_{i=k+1-\rho}^{k} \Upsilon^{T}(i) Q \Upsilon(i)-\sum_{i=k-\rho}^{k-1} \Upsilon^{T}(i) Q \Upsilon(i)\right]+\left[\sum_{i=k-d_{M}+1}^{k} x^{T}(i)(U \otimes T) x(i)-\sum_{i=k-d_{M}}^{k-1} x^{T}(i)(U \otimes T) x(i)\right]\right\} \\
& =\mathbb{E}\left\{\Upsilon^{T}(k) Q \Upsilon(k)-\Upsilon^{T}(k-\rho) Q \Upsilon(k-\rho)+x^{T}(k)(U \otimes T) x(k)-x^{T}\left(k-d_{M}\right)(U \otimes T) x\left(k-d_{M}\right)\right\}
\end{aligned}
$$

\section{$\mathbb{E}\left\{\Delta V_{5}(k)\right\}$}

$$
\begin{aligned}
&= \mathbb{E}\left\{V_{5}(k+1)-V_{5}(k)\right\} \\
&= \mathbb{E}\left\{\sum_{i=-\rho}^{-1}\left[\sum_{j=k+1+i}^{k} \eta^{T}(j)\left(U \otimes \hbar P_{2}\right) \eta(j)-\sum_{j=k+i}^{k-1} \eta^{T}(j)\left(U \otimes \hbar P_{2}\right) \eta(j)\right]\right. \\
&\left.+\sum_{i=-d_{M}}^{-r \rho-1}\left[\sum_{j=k+1+i}^{k} \eta^{T}(j)\left(U \otimes \hbar P_{2}\right) \eta(j)-\sum_{j=k+i}^{k-1} \eta^{T}(j)\left(U \otimes \hbar P_{2}\right) \eta(j)\right]\right\} \\
&=\mathbb{E}\left\{\rho \eta^{T}(k)\left(U \otimes \hbar P_{2}\right) \eta(k)-\sum_{j=k-\rho}^{k-1} \eta^{T}(j)\left(U \otimes \hbar P_{2}\right) \eta(j)+\left(d_{M}-r \rho\right) \eta^{T}(k)\left(U \otimes \hbar P_{2}\right) \eta(k)-\sum_{j=k-d_{M}}^{k-r \rho-1} \eta^{T}(j)\left(U \otimes \hbar P_{2}\right) \eta(j)\right\} \\
&=\mathbb{E}\left\{\eta^{T}(k)\left[U \otimes P_{2}\right] \eta(k)-\sum_{j=k-\rho}^{k-1} \eta^{T}(j)\left(U \otimes \hbar P_{2}\right) \eta(j)-\sum_{j=k-r \rho-h(k)}^{k-r \rho-1} \eta^{T}(j)\left(U \otimes \hbar P_{2}\right) \eta(j)-\sum_{j=k-d_{M}}^{k-1} \eta^{T}(j)\left(U \otimes \hbar P_{2}\right) \eta(j)\right\}
\end{aligned}
$$


In view of Lemma 4, we substitute (30)-(34) into (29) to get (36) shown at the bottom of the page.

From Assumption 1, we have

$$
\begin{aligned}
& {\left[\begin{array}{c}
x_{m}(k)-x_{\alpha}(k) \\
f\left(x_{m}(k)\right)-f\left(x_{\alpha}(k)\right)
\end{array}\right]^{T}} \\
& \quad \times\left[\begin{array}{cc}
-\left(F_{1}^{T} F_{2}+F_{2}^{T} F_{1}\right) & \left(F_{1}^{T}+F_{2}^{T}\right) \\
\left(F_{1}+F_{2}\right) & -2 I
\end{array}\right] \\
& \quad \times\left[\begin{array}{cc}
x_{m}(k)-x_{\alpha}(k) \\
f\left(x_{m}(k)\right)-f\left(x_{\alpha}(k)\right)
\end{array}\right] \geq 0
\end{aligned}
$$

that is

$$
\Lambda_{1}=:\left[\begin{array}{c}
\mathbf{x}_{m \alpha}(k) \\
\mathbf{f}_{m \alpha}(k)
\end{array}\right]^{T}\left[\begin{array}{cc}
-\breve{F}_{1} & \breve{F}_{2} \\
\breve{F}_{2}^{T} & -2 I
\end{array}\right]\left[\begin{array}{c}
\mathbf{x}_{m \alpha}(k) \\
\mathbf{f}_{m \alpha}(k)
\end{array}\right] \geq 0 .
$$

Similarly, we also have

$$
\begin{aligned}
\Lambda_{2}=: & {\left[\begin{array}{l}
\mathbf{x}_{m \alpha}(k-d(k)) \\
\mathbf{g}_{m \alpha}(k-d(k))
\end{array}\right]^{T}\left[\begin{array}{cc}
-\breve{G}_{1} & \breve{G}_{2} \\
\breve{G}_{2}^{T} & -2 I
\end{array}\right] } \\
& \times\left[\begin{array}{l}
\mathbf{x}_{m \alpha}(k-d(k)) \\
\mathbf{g}_{m \alpha}(k-d(k))
\end{array}\right] \geq 0
\end{aligned}
$$

$$
\Lambda_{3}=:\left[\begin{array}{l}
\mathbf{x}_{m \alpha}(k) \\
\mathbf{h}_{m \alpha}(k)
\end{array}\right]^{T}\left[\begin{array}{cc}
-\breve{H}_{1} & \breve{H}_{2} \\
\breve{H}_{2}^{T} & -2 I
\end{array}\right]\left[\begin{array}{l}
\mathbf{x}_{m \alpha}(k) \\
\mathbf{h}_{m \alpha}(k)
\end{array}\right] \geq 0
$$

Also, it follows from Assumption 2 and condition (15) that

$$
\begin{aligned}
\sigma_{m \alpha}^{T}(k)\left(P_{1}\right. & \left.+P_{2}\right) \sigma_{m \alpha}(k) \leq \lambda^{*}\left[\mathbf{x}_{m \alpha}(k) \mathcal{N}_{1}^{T} \mathcal{N}_{1} \mathbf{x}_{m \alpha}(k)\right. \\
& \left.+\mathbf{x}_{m \alpha}^{T}(k-d(k)) \mathcal{N}_{2}^{T} \mathcal{N}_{2} \mathbf{x}_{m \alpha}(k-d(k))\right] .
\end{aligned}
$$

Furthermore, according to the definition of $\eta(j)$ in (28), we let $x(k)$ be the trajectory of system (11). Then, it is not difficult to see that

$$
\begin{aligned}
x(k)-x(k-\rho) & =\sum_{j=k-\rho}^{k-1} \eta(j) \\
x(k-r \rho)-x(k-d(k)) & =\sum_{j=k-d(k)}^{k-r \rho-1} \eta(j) \\
x(k-d(k))-x\left(k-d_{M}\right) & =\sum_{j=k-d_{M}}^{k-r \rho-h(k)-1} \eta(j) .
\end{aligned}
$$

$$
\begin{aligned}
& \mathbb{E}\{\Delta V(k)\} \\
& \leq \sum_{1 \leq m<\alpha \leq N} \mathbb{E}\left\{\mathbf { x } _ { m \alpha } ^ { T } ( k ) \left[\left(A^{T} P_{1} A+\left(A-I_{n}\right)^{T} P_{2}\left(A-I_{n}\right)-P_{1}-N w_{m \alpha}^{(1,1)}\left[\Gamma_{1}^{T}\left(P_{1}+P_{2}\right) \Gamma_{1}+P_{1}+P_{2}\right]\right.\right.\right. \\
& \left.-N w_{m \alpha}^{(1)}\left[\Gamma_{1}^{T} P_{1} A+A^{T} P_{1} \Gamma_{1}+\Gamma_{1}^{T} P_{2}\left(A-I_{n}\right)+\left(A-I_{n}\right)^{T} P_{2} \Gamma_{1}\right]+\left(d_{M}-r \rho+1\right) S+T\right) \mathbf{x}_{m \alpha}(k) \\
& +2\left(A^{T} P_{1} A_{d}+\left(A-I_{n}\right)^{T} P_{2} A_{d}-N w_{m \alpha}^{(2)}\left(A^{T} P_{1}+\left(A-I_{n}\right)^{T} P_{2}\right) \Gamma_{2}\right. \\
& \left.-N w_{m \alpha}^{(1)} \Gamma_{1}^{T}\left(P_{1}+P_{2}\right) A_{d}-N w_{m \alpha}^{(1,2)} \Gamma_{1}^{T}\left(P_{1}+P_{2}\right) \Gamma_{2}\right) \mathbf{x}_{m \alpha}(k-d(k)) \\
& +2 \gamma\left(\left(A^{T} P_{1}+\left(A-I_{n}\right)^{T} P_{2}\right) B-N w_{m \alpha}^{(1)} \Gamma_{1}^{T}\left(P_{1}+P_{2}\right) B\right) \mathbf{f}_{m \alpha}(k) \\
& +2 \theta\left(\left(A^{T} P_{1}+\left(A-I_{n}\right)^{T} P_{2}\right) C-N w_{m \alpha}^{(1)} \Gamma_{1}^{T}\left(P_{1}+P_{2}\right) C\right) \mathbf{g}_{m \alpha}(k-d(k)) \\
& \left.+2\left(A^{T} P_{1}+\left(A-I_{n}\right)^{T} P_{2}-N w_{m \alpha}^{(1)} \Gamma_{1}^{T}\left(P_{1}+P_{2}\right)\right) \hat{\mathbf{h}}_{m \alpha}(k)\right] \\
& +\mathbf{x}_{m \alpha}^{T}(k-d(k))\left[\left(A_{d}^{T}\left(P_{1}+P_{2}\right) A_{d}-N w_{m \alpha}^{(2,2)}\left[\Gamma_{2}^{T}\left(P_{1}+P_{2}\right) \Gamma_{2}+P_{1}+P_{2}\right]\right.\right. \\
& \left.-N w_{m \alpha}^{(2)}\left[\Gamma_{2}^{T}\left(P_{1}+P_{2}\right) A_{d}+A_{d}^{T}\left(P_{1}+P_{2}\right) \Gamma_{2}\right]-S\right) \mathbf{x}_{m \alpha}(k-d(k)) \\
& +2 \gamma\left(A_{d}^{T}\left(P_{1}+P_{2}\right) B-N w_{m \alpha}^{(2)} \Gamma_{2}^{T}\left(P_{1}+P_{2}\right) B\right) \mathbf{f}_{m \alpha}(k) \\
& +2 \theta\left(A_{d}^{T}\left(P_{1}+P_{2}\right) C-N w_{m \alpha}^{(2)} \Gamma_{2}^{T}\left(P_{1}+P_{2}\right) C\right) \mathbf{g}_{m \alpha}(k-d(k)) \\
& \left.+2\left(A_{d}^{T}\left(P_{1}+P_{2}\right)-N w_{m \alpha}^{(2)} \Gamma_{2}^{T}\left(P_{1}+P_{2}\right)\right) \hat{\mathbf{h}}_{m \alpha}(k)\right] \\
& +\gamma \mathbf{f}_{m \alpha}^{T}(k)\left[B^{T}\left(P_{1}+P_{2}\right) B \mathbf{f}_{m \alpha}(k)+2 \theta B^{T}\left(P_{1}+P_{2}\right) C \mathbf{g}_{m \alpha}(k-d(k))+2 B^{T}\left(P_{1}+P_{2}\right) \hat{\mathbf{h}}_{m \alpha}(k)\right] \\
& +\theta \mathbf{g}_{m \alpha}^{T}(k-d(k))\left[C^{T}\left(P_{1}+P_{2}\right) C \mathbf{g}_{m \alpha}(k-d(k))+2 C^{T}\left(P_{1}+P_{2}\right) \hat{\mathbf{h}}_{m \alpha}(k)\right] \\
& +\tilde{\mu} \mathbf{h}_{m \alpha}^{T}(k) R \mathbf{h}_{m \alpha}(k)+\hat{\mathbf{h}}_{m \alpha}^{T}(k)\left(P_{1}+P_{2}-\frac{1}{\tilde{\mu}} R\right) \hat{\mathbf{h}}_{m \alpha}(k)-\mathbf{x}_{m \alpha}^{T}\left(k-d_{M}\right) T \mathbf{x}_{m \alpha}\left(k-d_{M}\right) \\
& +\Upsilon_{m \alpha}^{T}(k) \bar{Q} \Upsilon_{m \alpha}(k)-\Upsilon_{m \alpha}^{T}(k-\rho) \bar{Q} \Upsilon_{m \alpha}(k-\rho)+\sigma_{m \alpha}^{T}(k)\left(P_{1}+P_{2}\right) \sigma_{m \alpha}(k)-\sum_{j=k-\rho}^{k-1} \hbar \eta_{m \alpha}^{T}(j) P_{2} \eta_{m \alpha}(j) \\
& \left.-\sum_{j=k-r \rho-h(k)}^{k-r \rho-1} \hbar \eta_{m \alpha}^{T}(j) P_{2} \eta_{m \alpha}(j)-\sum_{j=k-d_{M}}^{k-r \rho-h(k)-1} \hbar \eta_{m \alpha}^{T}(j) P_{2} \eta_{m \alpha}(j)\right\}
\end{aligned}
$$


Based on the above equalities (41)-(43), the following equations hold for any matrices $X_{l}, Y_{l}$, and $Z_{l}(l=1,2, \ldots, r+7)$, with appropriate dimensions:

$$
\begin{aligned}
& 0=2 \delta^{T}(k) X\left[x(k)-x(k-\rho)-\sum_{j=k-\rho}^{k-1} \eta(j)\right] \\
& 0=2 \delta^{T}(k) Y\left[x(k-r \rho)-x(k-d(k))-\sum_{j=k-d(k)}^{k-r \rho-1} \eta(j)\right] \\
& 0=2 \delta^{T}(k) Z\left[x(k-d(k))-x\left(k-d_{M}\right)-\sum_{j=k-d_{M}}^{k-r \rho-h(k)-1} \eta(j)\right]
\end{aligned}
$$

with $X=\operatorname{col}\left\{U \otimes X_{1}, U \otimes X_{2}, \ldots, U \otimes X_{r+7}\right\}$, $Y=\operatorname{col}\left\{U \otimes Y_{1}, U \otimes Y_{2}, \ldots, U \otimes Y_{r+7}\right\}$, and $Z=$ $\operatorname{col}\left\{U \otimes Z_{1}, U \otimes Z_{2}, \ldots, U \otimes Z_{r+7}\right\}$.

Using Lemma 4, formulas (44)-(46) can be converted into the following:

$$
\begin{aligned}
0= & \sum_{1 \leq m<\alpha \leq N} 2 \delta_{m \alpha}^{T}(k) \mathcal{X} \\
& \times\left[\mathbf{x}_{m \alpha}(k)-\mathbf{x}_{m \alpha}(k-\rho)-\sum_{j=k-\rho}^{k-1} \eta_{m \alpha}(j)\right] \\
0= & \sum_{1 \leq m<\alpha \leq N} 2 \delta_{m \alpha}^{T}(k) \mathcal{Y} \\
& \times\left[\mathbf{x}_{m \alpha}(k-r \rho)-\mathbf{x}_{m \alpha}(k-d(k))-\sum_{j=k-d(k)}^{k-r \rho-1} \eta_{m \alpha}(j)\right] \\
0= & \sum_{1 \leq m<\alpha \leq N} 2 \delta_{m \alpha}^{T}(k) \mathcal{Z} \\
& \times\left[\mathbf{x}_{m \alpha}(k-d(k))-\mathbf{x}_{m \alpha}\left(k-d_{M}\right)-\sum_{j=k-d_{M}}^{k-r \rho-h(k)-1} \eta_{m \alpha}(j)\right] .
\end{aligned}
$$

On the other hand, for any appropriately dimensioned matrices $M=M^{T} \geq 0$ and $J=J^{T} \geq 0$, the following equations are true:

$$
\begin{aligned}
& 0=\sum_{j=k-\rho}^{k-1} \delta^{T}(k)\left(I_{r+7} \otimes(U \otimes M)\right) \delta(k) \\
& -\sum_{j=k-\rho}^{k-1} \delta^{T}(k)\left(I_{r+7} \otimes(U \otimes M)\right) \delta(k) \\
& =\rho \delta^{T}(k)\left(I_{r+7} \otimes(U \otimes M)\right) \delta(k) \\
& -\sum_{j=k-\rho}^{k-1} \delta^{T}(k)\left(I_{r+7} \otimes(U \otimes M)\right) \delta(k) \\
& =\sum_{1 \leq m<\alpha \leq N}\left\{\rho \delta_{m \alpha}^{T}(k)\left(I_{r+7} \otimes M\right) \delta_{m \alpha}(k)\right. \\
& \left.-\sum_{j=k-\rho}^{k-1} \delta_{m \alpha}^{T}(k)\left(I_{r+7} \otimes M\right) \delta_{m \alpha}(k)\right\}
\end{aligned}
$$

$$
\begin{aligned}
& 0=\sum_{j=k-d_{M}}^{k-r \rho-1} \delta^{T}(k)\left(I_{r+7} \otimes(U \otimes J)\right) \delta(k) \\
& -\sum_{j=k-d_{M}}^{k-r \rho-1} \delta^{T}(k)\left(I_{r+7} \otimes(U \otimes J)\right) \delta(k) \\
& =\left(d_{M}-d_{m}\right) \delta^{T}(k)\left(I_{r+7} \otimes(U \otimes J)\right) \delta(k) \\
& -\sum_{j=k-r \rho-h(k)}^{k-r \rho-1} \delta^{T}(k)\left(I_{r+7} \otimes(U \otimes J)\right) \delta(k) \\
& -\sum_{j=k-d_{M}}^{k-r \rho-h(k)-1} \delta^{T}(k)\left(I_{r+7} \otimes(U \otimes J)\right) \delta(k) \\
& =\sum_{1 \leq m<\alpha \leq N}\left\{\left(d_{M}-d_{m}\right) \delta_{m \alpha}^{T}(k)\left(I_{r+7} \otimes J\right) \delta_{m \alpha}(k)\right. \\
& -\sum_{j=k-r \rho-h(k)}^{k-r \rho-1} \delta_{m \alpha}^{T}(k)\left(I_{r+7} \otimes J\right) \delta_{m \alpha}(k) \\
& \left.-\sum_{j=k-d_{M}}^{k-r \rho-h(k)-1} \delta_{m \alpha}^{T}(k)\left(I_{r+7} \otimes J\right) \delta_{m \alpha}(k)\right\} .
\end{aligned}
$$

Then, when (37)-(40) and (47)-(51) are all added into (36), we can derive that

$$
\begin{aligned}
& \mathbb{E}\{\Delta V(k)\} \\
& \leq \sum_{1 \leq m<\alpha \leq N} \mathbb{E}\left\{\delta_{m \alpha}^{T}(k)\left(\Pi_{1}+\Pi_{2}+\bar{\Pi}_{3}+\Pi_{4}+\Pi_{4}^{T}\right)\right. \\
& \times \delta_{m \alpha}(k)+\sum_{l=1}^{3} \varrho_{l} \Lambda_{l} \\
& -\sum_{j=k-\rho}^{k-1} \zeta_{m \alpha}^{T}(k, j) \Xi_{1} \zeta_{m \alpha}(k, j) \\
& -\sum_{j=k-d(k)}^{k-r \rho-1} \zeta_{m \alpha}^{T}(k, j) \Xi_{2} \zeta_{m \alpha}(k, j) \\
& \left.-\sum_{j=k-d_{M}}^{k-r \rho-h(k)-1} \zeta_{m \alpha}^{T}(k, j) \Xi_{3} \zeta_{m \alpha}(k, j)\right\} \\
& =\sum_{1 \leq m<\alpha \leq N} \mathbb{E}\left\{\delta_{m \alpha}^{T}(k) \Pi_{m \alpha} \delta_{m \alpha}(k)\right. \\
& -\sum_{j=k-\rho}^{k-1} \zeta_{m \alpha}^{T}(k, j) \Xi_{1} \zeta_{m \alpha}(k, j) \\
& -\sum_{j=k-d(k)}^{k-r \rho-1} \zeta_{m \alpha}^{T}(k, j) \Xi_{2} \zeta_{m \alpha}(k, j) \\
& \left.-\sum_{j=k-d_{M}}^{k-r \rho-h(k)-1} \zeta_{m \alpha}^{T}(k, j) \Xi_{3} \zeta_{m \alpha}(k, j)\right\}
\end{aligned}
$$

where $\zeta_{m \alpha}(k, j)=\operatorname{col}\left\{\delta_{m \alpha}(k), \eta_{m \alpha}(j)\right\}$ 
Now, from (16) and (17) in Theorem 1, we have $\Xi_{l} \geq 0(l=1,2,3)$ and $\Pi_{m \alpha}<0$, where $\delta_{m \alpha}^{T}(k) \bar{\Pi}_{3} \delta_{m \alpha}(k)=$ $\delta_{m \alpha}^{T}(k) \Pi_{3} \delta_{m \alpha}(k)+\sum_{l=1}^{3} \varrho_{l} \Lambda_{l}$. Then it follows readily that $\mathbb{E}\{V(k+1)-V(k)\} \leq \chi \sum_{1 \leq m<\alpha \leq N}\left|\delta_{m \alpha}(k)\right|^{2}$ where $\chi=\max _{1 \leq m<\alpha \leq N}\left\{\lambda_{\max }\left(\Pi_{m \alpha}\right)\right\}$. Noticing that $\chi<0$ and $\left|\mathbf{x}_{m \alpha}(k)\right|^{2} \leq\left|\bar{\delta}_{m \alpha}(k)\right|^{2}$, it is not difficult to deduce that

$$
\mathbb{E}\{V(k+1)\}-\mathbb{E}\{V(k)\} \leq \chi \sum_{1 \leq m<\alpha \leq N}\left|\mathbf{x}_{m \alpha}(k)\right|^{2} .
$$

Letting $k_{0}$ be an arbitrary positive integer, it implies from (53) that

$$
\begin{aligned}
\mathbb{E}\left\{V\left(k_{0}+1\right)\right\}-\mathbb{E}\{V(1)\} & =\sum_{k=1}^{k_{0}} \mathbb{E}\{V(k)\} \\
& \leq \chi \sum_{k=1}^{k_{0}} \sum_{1 \leq m<\alpha \leq N}\left|\mathbf{x}_{m \alpha}(k)\right|^{2} .
\end{aligned}
$$

Consequently, with condition (6), we obtain

$$
\sum_{k=1}^{k_{0}} \sum_{1 \leq m<\alpha \leq N}\left|\mathbf{x}_{m \alpha}(k)\right|^{2} \leq-\frac{1}{\chi} \mathbb{E}\{V(1)\}<+\infty .
$$

Inequality (55) indicates that the series $\sum_{1<m<\alpha<N}\left|\mathbf{x}_{m \alpha}(k)\right|^{2} \rightarrow 0$ as $k_{0} \rightarrow+\infty$. That is, $\lim _{k \rightarrow+\infty}\left|x_{m}(k)-x_{\alpha}(k)\right|^{2}=0(1 \leq m<\alpha \leq N)$. This completes the proof of Theorem 1 .

\section{REFERENCES}

[1] M. Aldana, "Boolean dynamics of networks with scale-free topology," Physica D, vol. 185, pp. 45-66, 2003.

[2] F. M. Atay, T. Biyikoglu, and J. Jost, "Network synchronization: Spectral versus statistical properties," Physica D, vol. 224, no. 1-2, pp. 35-41, 2006.

[3] I. V. Belykh, V. N. Belykh, and M. Hasler, "Blinking model and synchronization in small-world networks with a time-varying coupling," Physica D, vol. 195, no. 1-2, pp. 188-206, 2004.

[4] S. Boyd, L. E. Ghaoui, E. Feron, and V. Balakrishnan, Linear Matrix Inequalities in System and Control Theory. Philadelphia, PA: SIAM, 1994.

[5] L. Chen, C. Qiu, and H. B. Huang, "Synchronization with on-off coupling: Role of time scales in network dynamics," Phys. Rev. E, Stat. Phys. Plasmas Fluids Relat. Interdiscip. Top., vol. 79, no. 4, Article 045101.

[6] C. Dangalchev, "Generation models for scale-free networks," Physica $A$, vol. 338, pp. 659-671, 2004.

[7] H. Gao and T. Chen, "New results on stability of discrete-time systems with time-varying state delay," IEEE Trans. Autom. Control, vol. 52, no. 2, pp. 328-334, Feb. 2007.

[8] Y. He, G. Liu, D. Rees, and M. Wu, " $H_{\infty}$ filtering for discrete-time systems with time-varying delay," Signal Process., DOI: 10.1016/j. sigpro.2008.08.008, to be published.

[9] S. Kauffman, C. Peterson, B. Samuelsson, and C. Troein, "Random Boolean network models and the yeast transcriptional network," Proc. Nat. Acad. Sci. USA, vol. 100, no. 25, pp. 14796-14799, 2003.

[10] R. Z. Khasminskii, "Stochastic stability of differential equations," Alphen aan den Rijn, Sijthoffand Noor, Khasminskiidhoff, 1980.

[11] H. K. Khalil, Nonlinear Systems. Upper Saddle River, NJ: PrenticeHall, 1996.

[12] A. N. Langville and W. J. Stewart, "The Kronecker product and stochastic automata networks," J. Comput. Appl. Math., vol. 167, pp. 429-447, 2004.

[13] C. P. Li, W. G. Sun, and J. Kurths, "Synchronization of complex dynamical networks with time delays," Physica A, vol. 361, pp. 24-34, 2006.
[14] Z. Li and G. Chen, "Global synchronization and asymptotic stability of complex dynamical networks," IEEE Trans. Circuits Syst. II, Reg. Papers, vol. 53, no. 1, pp. 28-33, Jan. 2006.

[15] J. Liang, Z. Wang, Y. Liu, and X. Liu, "Global synchronization control of general delayed discrete-time networks with stochastic coupling and disturbances," IEEE Trans. Syst. Man Cybern. B, Cybern., vol. 38, no. 4, pp. 1073-1083, Aug. 2008.

[16] X. Liu and T. Chen, "Exponential synchronization of nonlinear coupled dynamical networks with a delayed coupling," Physica A, vol. 381, pp. 82-92, 2007.

[17] Y. Liu, Z. Wang, and X. Liu, "Global exponential stability of generalized recurrent neural networks with discrete and distributed delays," Neural Netw., vol. 19, no. 5, pp. 667-675, 2006.

[18] Y. Liu, Z. Wang, and X. Liu, "Exponential synchronization of complex networks with Markovian jump and mixed delays," Phys. Lett. A, vol. 372, pp. 3986-3998, 2008.

[19] Y. Liu, Z. Wang, J. Liang, and X. Liu, "Synchronization and state estimation for discrete-time complex networks with distributed delays," IEEE Trans. Syst. Man Cybern. B, Cybern., vol. 38, no. 5, pp. 1314-1325, Oct. 2008.

[20] W. Lu and T. Chen, "Synchronization analysis of linearly coupled networks of discrete time systems," Physica D, vol. 198, pp. 148-168, 2004.

[21] W. Lu and T. Chen, "Global synchronization of discrete-time dynamical network with a directed graph," IEEE Trans. Circuits Syst. II, Exp. Briefs, vol. 54, no. 2, pp. 136-140, Feb. 2007.

[22] Y. Lv, W. Lv, and J. Sun, "Convergence dynamics of stochastic reaction-diffusion recurrent neural networks with continuously distributed delays," Nonlinear Anal., Real World Appl., vol. 9, pp. 1590-1606, 2008.

[23] X. Mao, "Exponential stability of stochastic delay interval systems with Markovian switching," IEEE Trans. Autom. Control, vol. 47, no. 10, pp. 1604-1612, Oct. 2002.

[24] S. Mou, H. Gao, W. Qiang, and K. Chen, "New delay-dependent exponential stability for neural networks with time delay," IEEE Trans. Syst. Man Cybern. B, Cybern., vol. 38, no. 2, pp. 571-576, Apr. 2008.

[25] S. Mou, Y. Zhao, H. Gao, and W. Qiang, "Further improvement on synchronization stability of complex networks with coupling delays," Int. J. Comput. Math., vol. 85, no. 8, pp. 1255-1263, 2008.

[26] M. Porfiri, D. J. Stilwell, E. M. Bollt, and J. D. Skufca, "Random talk: Random walk and synchronizability in a moving neighborhood network," Physica D, vol. 224, no. 1-2, pp. 102-113, 2006.

[27] A. Ribeiro, R. Zhu, and S. A. Kauffman, "A general modeling strategy for gene regulatory networks with stochastic dynamics," J. Comput. Biol., vol. 13, no. 9, pp. 1630-1639, 2006.

[28] Y. Shen and J. Wang, "Noise-induced stabilization of the recurrent neural networks with mixed time-varying delays and Markovian-switching parameters," IEEE Trans. Neural Netw., vol. 18, no. 6, pp. 1857-1862, Nov. 2007.

[29] S. Strogatz, "Exploring complex networks," Nature, vol. 410, pp. 268-276, 2001.

[30] Y. Suemitsu and S. Nara, "A note on time delayed effect in a recurrent neural network model," Neural Comput. Appl., vol. 11, no. 3-4, pp. 137-143, 2003.

[31] Z. Toroczkai, "Complex networks: The challenge of interaction topology," Los Alamos Sci., vol. 29, pp. 94-109, 2005.

[32] X. Wang and G. Chen, "Complex networks: Small-world, scale-free, and beyond," IEEE Circuits Syst. Mag., vol. 3, no. 1, pp. 6-20, 2003.

[33] Z. Wang, F. Yang, and X. Liu, "Robust filtering for systems with stochastic nonlinearities and deterministic uncertainties," Proc. IMechE/J. Syst. Control Eng., vol. 220, no. 3, pp. 171-182, 2006.

[34] W. Xiong, D. W. C. Ho, and J. Cao, "Synchronization analysis of singular hybrid coupled networks," Phys. Lett. A, vol. 372, no. 44, pp. 6633-6637, 2008.

[35] J. Yao, H. O. Wang, Z.-H. Guan, and W. Xu, "Passive stability and synchronization of complex spatio-temporal switching networks with time delays," Automatica, vol. 45, no. 7, pp. 1721-1728, Jul. 2009.

[36] D. Yue, Y. Zhang, E. Tian, and C. Peng, "Delay-distribution-dependent exponential stability criteria for discrete-time recurrent neural networks with stochastic delay," IEEE Trans. Neural Netw., vol. 19, no. 7, pp. 1299-1306, Jul. 2008 .

[37] H. Zhang and Y. Wang, "Stability analysis of Markovian jumping stochastic Cohen-Grossberg neural networks with mixed time delays," IEEE Trans. Neural Netw., vol. 19, no. 2, pp. 366-370, Feb. 2008.

[38] Y. Zhao, H. Gao, J. Lam, and B. Du, "Stability and stabilization of delayed T-S fuzzy systems: A delay partitioning approach," IEEE Trans. Fuzzy Syst., vol. 17, no. 4, pp. 750-762, Aug. 2008. 


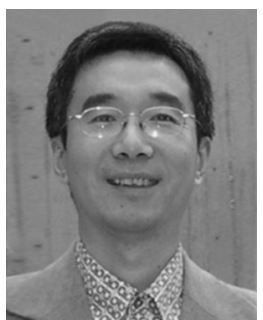

Zidong Wang (SM'03) was born in Jiangsu, China, in 1966. He received the B.Sc. degree in mathematics from Suzhou University, Suzhou, China, in 1986 and the M.Sc. degree in applied mathematics and the $\mathrm{Ph} . \mathrm{D}$. degree in electrical and computer engineering from Nanjing University of Science and Technology, Nanjing, China, in 1990 and 1994, respectively.

Currently, he is a Professor of Dynamical Systems and Computing at Brunel University, Uxbridge, U.K. His research interests include dynamical systems, signal processing, bioinformatics, control theory, and applications. He has published more than 120 papers in refereed international journals.

Dr. Wang is an Associate Editor for 12 international journals including the IEEE TRANSACTIONS ON AUTOMATIC CONTROL, the IEEE TRANSACTIONS on Neural Networks, the IEEE Transactions on Signal Processing, the IEEE Transactions on Systems, Man, And Cybernetics-PART C: APPLICATIONS AND REVIEWS, and the IEEE TRANSACTIONS ON CONTROL SYSTEMS TECHNOLOGY.

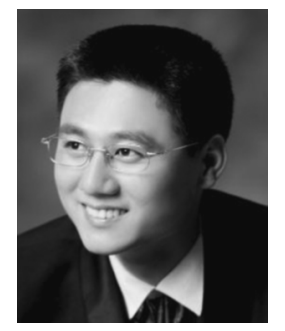

Yao Wang received the B.Sc. and M.Sc. degrees in applied mathematics from the Department of Mathematics, Yangzhou University, Yangzhou, China, in 2004 and 2007, respectively. Currently, he is working towards the Ph.D. degree at the School of Information Science and Technology, Donghua University, Shanghai, China.

His research interests include dynamical systems, complex networks, systems biology, stochastic systems, and time-delay systems. He is a very active reviewer for many international journals.

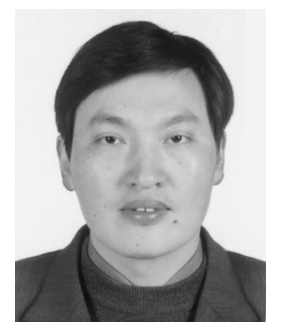

Yurong Liu received the B.Sc. degree in mathematics from Suzhou University, Suzhou, China, in 1986, the M.Sc. degree in applied mathematics from Nanjing University of Science and Technology, Nanjing, China, in 1989, and the Ph.D. degree in applied mathematics from Suzhou University, Suzhou, China, in 2000.

Currently, he is a Professor at the Department of Mathematics, Yangzhou University, Yangzhou, China. His current interests include neural networks, nonlinear dynamics, time-delay systems, and chaotic

dynamics. 